Article

\title{
BeiDou Inter-Satellite-Type Bias Evaluation and Calibration for Mixed Receiver Attitude Determination
}

\author{
Nandakumaran Nadarajah ${ }^{1, *}$, Peter J. G. Teunissen ${ }^{1,2}$ and Noor Raziq ${ }^{1}$ \\ ${ }^{1}$ GNSS Research Centre, Department of Spatial Sciences, Curtin University, GPO Box U1987, Perth, \\ WA 6845, Australia; E-Mails: p.teunissen@curtin.edu.au (P.J.G.T.); noor.raziq@ curtin.edu.au (N.R.) \\ ${ }^{2}$ Delft Institute of Earth Observation and Space Systems (DEOS), Delft University of Technology, \\ PO Box 5058, 2600 GB Delft, The Netherlands
}

* Author to whom correspondence should be addressed; E-Mail: n.nadarajah@curtin.edu.au.

Received: 12 June 2013; in revised form: 12 July 2013 / Accepted: 16 July 2013 /

Published: 22 July 2013

\begin{abstract}
The Chinese BeiDou system (BDS), having different types of satellites, is an important addition to the ever growing system of Global Navigation Satellite Systems (GNSS). It consists of Geostationary Earth Orbit (GEO) satellites, Inclined Geosynchronous Satellite Orbit (IGSO) satellites and Medium Earth Orbit (MEO) satellites. This paper investigates the receiver-dependent bias between these satellite types, for which we coined the name "inter-satellite-type bias" (ISTB), and its impact on mixed receiver attitude determination. Assuming different receiver types may have different delays/biases for different satellite types, we model the differential ISTBs among three BeiDou satellite types and investigate their existence and their impact on mixed receiver attitude determination. Our analyses using the real data sets from Curtin's GNSS array consisting of different types of BeiDou enabled receivers and series of zero-baseline experiments with BeiDou-enabled receivers reveal the existence of non-zero ISTBs between different BeiDou satellite types. We then analyse the impact of these biases on BeiDou-only attitude determination using the constrained (C-)LAMBDA method, which exploits the knowledge of baseline length. Results demonstrate that these biases could seriously affect the integer ambiguity resolution for attitude determination using mixed receiver types and that a priori correction of these biases will dramatically improve the success rate.
\end{abstract}


Keywords: Global Navigation Satellite Systems (GNSS); BeiDou system (BDS); inter-satellite-type biases; attitude determination; multivariate constrained integer leastsquares(MC-LAMBDA); carrier phase ambiguity resolution

\section{Introduction}

The Chinese BeiDou System (BDS), having different types of satellites, is an important addition to the ever growing system of Global Navigation Satellite Systems (GNSS). The BDS currently under development will consist of five Geostationary Earth Orbit (GEO) satellites, three Inclined Geosynchronous Satellite Orbit (IGSO) satellites and twenty-seven Medium Earth Orbit (MEO) satellites [1,2]. Presently, it consists of five GEO, five IGSO and four MEO satellites transmitting navigation signals in quadrature phase-shift keying (QPSK) modulation on a total of three frequency bands (B1, B2, B3). This paper investigates the receiver-dependent bias between these satellite types, for which we coined the name "inter-satellite-type bias" (ISTB), and its impact on mixed receiver attitude determination.

Having 14 fully operational satellites, BDS has already had the standalone capability of satellite-based positioning, navigation and timing (PNT) solutions, at least in the Asia-Pacific region. Analyses of BeiDou-based PNT solutions have been reported in various studies. Apart from simulation-based studies in [3-7], analyses of BeiDou PNT with real data have been reported in [8-18]. Measurement quality and relative positioning analyses with real data collected using Chinese GNSS receivers (UB240-CORS) are reported in [10,19]. Precise point positioning results with the same receiver type are reported in [15]. Initial assessment of real data collected using non-Chinese GNSS receivers and with post-processed orbit and clock products [20,21] independent of the control segment is reported in [8]. The same products are used to analyse precise point positioning in [11,14] and triple-frequency relative positioning in [9]. Similar products are used in [12] to analyse the contribution of BeiDou in single point positioning. With the recent release of BeiDou interface control document (ICD) [1], one can expect increased research on BeiDou based-PNT solutions outside China.

Multiple GNSS receivers/antennas rigidly mounted on a platform can be used to determine platform attitude (orientation) (see, for example [22-28]). GNSS-based attitude determination offers several advantages, including that it is not affected by drift, is lower in cost and requires less maintenance than traditional methods. Precise attitude determination, however, relies on successful resolution of the integer carrier phase ambiguities. The least squares ambiguity decorrelation adjustment (LAMBDA) method [29] is currently the standard method for solving unconstrained and linearly-constrained GNSS ambiguity resolution problems (see, for example [30-37]). For such models, the method is known to be numerically efficient and optimal in the sense that it provides integer ambiguity solutions with the highest possible success-rate [38-40]. To exploit the known baseline length, we make use of the constrained (C-)LAMBDA method [41-49]. BeiDou-based attitude determination using identical receivers has been analysed in [16-18]. 
In this paper, we consider mixed receiver attitude determination using single- and dual-frequency BeiDou observables. Assuming different receiver types may have different delays/biases for different satellite types, we model the differential ISTBs among three BeiDou satellite types. We develop an extended GNSS model, taking into account these biases, and describe the estimation of these biases. Our analyses using the data from two real data campaigns (one is with Curtin's permanent GNSS array consisting of different types of BeiDou-enabled receivers, and the other is with zero-baseline using BeiDou enabled receivers) reveal the existence of non-zero ISTBs between different BeiDou satellite types. We observe that these biases are stable and constant. Hence, we use a priori estimated biases to correct BeiDou observations, so that one can use classical double difference processing without loosing redundancy. We then analyse the impact of these biases on BeiDou-only attitude determination using the constrained (C-)LAMBDA method, which exploits the knowledge of baseline length. Results demonstrate that ISTBs could seriously affect the integer ambiguity resolution for attitude determination using mixed receiver types and that a priori correction of these biases will dramatically improve the success rate.

This contribution is organized as follows. Section 2 describes the functional and stochastic model for BeiDou observations, with special attention to ISTBs and associated processing approaches. Section 3 formulates the quadratically-constrained BeiDou model for attitude determination and describes the C-LAMBDA method. Section 4 demonstrates the existence of non-zero ISTBs between different BeiDou satellite types using real data and presents the results of attitude determination, revealing the impact of ISTBs. Finally, Section 5 contains the summary and conclusions of this contribution.

\section{BeiDou Observations}

This section presents the BeiDou observation model, distinguishing satellite types, namely GEO, IGSO and MEO, to accommodate receiver-dependent delays (biases) for different satellite types. Since the BeiDou system uses the code division multiple access (CDMA) technique, the code and phase observations of receiver $r$ tracking satellite $s_{\beta}$ of type $\beta$ at frequency $j$, denoted by $p_{r, j}^{s_{\beta}}$ and $\phi_{r, j}^{s_{\beta}}$, respectively, are given as [50]:

$$
\begin{aligned}
& p_{r, j}^{s_{\beta}}=\rho_{r}^{s_{\beta}}+d t_{r}-d t^{s_{\beta}}+a_{r, j}^{s_{\beta}}+d_{r, j}^{, \beta}-d_{, j}^{s_{\beta}}+e_{r, j}^{s_{\beta}}, \quad j=1, \ldots, f ; \quad s_{\beta}=1, \ldots, m_{\beta} ; \beta=1, \ldots, \eta \\
& \phi_{r, j}^{s_{\beta}}=\rho_{r}^{s_{\beta}}+d t_{r}-d t^{s_{\beta}}+\alpha_{r, j}^{s_{\beta}}+\lambda_{j}\left(\delta_{r, j}^{, \beta}+\varphi_{r, j}-\delta_{, j}^{s_{\beta}}-\varphi_{, j}^{s_{\beta}}+N_{r, j}^{s_{\beta}}\right)+\epsilon_{r, j}^{s_{\beta}}
\end{aligned}
$$

with $f$ the number of frequencies, $m_{\beta}$ the number of type- $\beta$ satellites tracked, $\eta$ the number of satellite types, $\rho_{r}^{s_{\beta}}$ the topocentric distance between receiver $r$ and satellite $s_{\beta}, d t_{r}$ the receiver clock error, $d t^{s_{\beta}}$ the satellite clock error, $a_{r, j}^{s_{\beta}}$ the (frequency-dependent) code atmospheric delay, $\alpha_{r, j}^{s_{\beta}}$ the (frequency-dependent) phase atmospheric delay, $d_{r, j}^{, \beta}$ the (satellite-type-dependent) hardware code delay in the receiver, $d_{, j}^{s_{\beta}}$ the hardware code delay in the satellite, $\lambda_{j}$ the wavelength, $\delta_{r, j}^{, \beta}$ the (satellite-type-dependent) hardware phase delay in the receiver, $\varphi_{r, j}$ the initial phase in the receiver, $\delta_{, j}^{s_{\beta}}$ the hardware phase delay in the satellite, $\varphi_{, j}^{s_{\beta}}$ the initial phase in the satellite, $N_{r, j}^{s_{\beta}}$ the (integer) phase ambiguities, $e_{r, j}^{s_{\beta}}$ all other code errors, including measurement noise, and $\epsilon_{r, j}^{s_{\beta}}$ all other phase errors, including measurement noise. For simplicity of formulation, we assume that satellites are ordered, such that the first $m_{1}$ satellites are of type 1 , the next $m_{2}$ satellites are of type 2 , and so on 
( $\sum_{\beta=1}^{\eta} m_{\beta}=m$, the number of tracked satellites). Note that all variables are expressed in meters; except the satellite-type-dependent biases of phase observations and the ambiguity, which are expressed in cycles.

The between-receiver single difference (SD) pseudo-range and carrier-phase observations of two BeiDou receivers, $r$ and 1 , at frequency $j$ from satellite $s_{\beta}$ of type $\beta$, denoted as $p_{1 r}^{s_{\beta}}$ and $\phi_{1 r}^{s_{\beta}}$, respectively, are given as:

$$
\begin{aligned}
& p_{1 r, j}^{s_{\beta}}=p_{r, j}^{s_{\beta}}-p_{1, j}^{s_{\beta}}=\rho_{1 r}^{s_{\beta}}+d t_{1 r}+a_{1 r, j}^{s_{\beta}}+d_{1 r, j}^{, \beta}+e_{1 r, j}^{s_{\beta}} \\
& \phi_{1 r, j}^{s_{\beta}}=\phi_{r, j}^{s_{\beta}}-\phi_{1, j}^{s_{\beta}}=\rho_{1 r}^{s_{\beta}}+d t_{1 r}+\alpha_{1 r, j}^{s_{\beta}}+\lambda_{j}\left(\delta_{1 r, j}^{, \beta}+\varphi_{1 r, j}+N_{1 r, j}^{s_{\beta}}\right)+\epsilon_{1 r, j}^{s_{\beta}}
\end{aligned}
$$

where the satellite-specific biases are eliminated. Attitude determination for a small platform considered in this paper is based on multiple BeiDou receivers forming very short-baselines for which the differential atmospheric delays can be ignored, that is, $a_{1 r, j}^{s_{\beta}}=\alpha_{1 r, j}^{s_{\beta}}=0 \forall j, s_{\beta}$.

Further differencing the above SD observables between satellites eliminates the receiver-dependent biases: with the first satellite of the satellite-type 1 as the pivot, the double difference (DD) observables are given as:

$$
\begin{aligned}
& p_{1 r, j}^{1_{1} s_{\beta}}=p_{1 r, j}^{s_{\beta}}-p_{1 r, j}^{1_{1}}=\rho_{1 r}^{1_{1} s_{\beta}}+d_{1 r, j}^{1 \beta}+e_{1 r, j}^{1_{1} s_{\beta}} \\
& \phi_{1 r, j}^{1_{1} s_{\beta}}=\phi_{1 r, j}^{s_{\beta}}-\phi_{1 r, j}^{1_{1}}=\rho_{1 r}^{1_{1} s_{\beta}}+\lambda_{j}\left(\delta_{1 r, j}^{1 \beta}+N_{1 r, j}^{1 s_{\beta}}\right)+\epsilon_{1 r, j}^{1_{1} s_{\beta}}
\end{aligned}
$$

In classical double differencing for single satellite-type systems, such as GPS, the terms, $d_{1 r, j}^{1 \beta}$ and $\delta_{1 r, j}^{, 1 \beta}$, do not exist. However, as shown in Section 4.2, there may exist non-zero differential inter-satellite-type biases (ISTBs) for the BeiDou system if one uses mixed receiver types. That is, $\delta_{1 r, j}^{1 \beta} \neq 0$ and $d_{1 r, j}^{1 \beta} \neq 0$ for $\beta \neq 1$.

The linearized DD observation equations corresponding to Equations (5) and (6) read:

$$
\begin{aligned}
& \mathrm{E}\left(\Delta p_{1 r, j}^{1_{1} s_{\beta}}\right)=g_{r}^{1_{1} s_{\beta} T} b+d_{1 r, j}^{, 1 \beta}, \\
& \mathrm{E}\left(\Delta \phi_{1 r, j}^{1_{1} s_{\beta}}\right)=g_{r}^{1_{1} s_{\beta} T} b+\lambda_{j}\left(\delta_{1 r, j}^{, 1 \beta}+N_{1 r, j}^{1_{1} s_{\beta}}\right)
\end{aligned}
$$

where $\mathrm{E}(\cdot)$ denote the expectation operator, $\Delta p_{1 r, j}^{1_{1} s_{\beta}}$ and $\Delta \phi_{1 r, j}^{1_{1} s_{\beta}}$ are the observed-minus-computed code and phase observations, $b$ is the baseline vector containing relative position components and $g_{r}^{11_{1} s_{\beta}}$ is the geometry vector given as $g_{r}^{1_{1} s_{\beta}}=u_{r}^{1_{1}}-u_{r}^{s_{\beta}}$, with $u_{r}^{s_{\beta}}$ the unit line-of-sight vector from receiver $r$ to satellite $s_{\beta}$.

The vectorial forms of the DD observation equations for the $j$ th frequency read:

$$
\begin{aligned}
& \mathrm{E}\left(y_{p ; j}\right)=G_{r} b+H_{\eta} h_{p ; j} \\
& \mathrm{E}\left(y_{\phi ; j}\right)=G_{r} b+\lambda_{j} z_{j}+\lambda_{j} H_{\eta} h_{\phi ; j}
\end{aligned}
$$


with:

$$
\begin{aligned}
y_{p ; j} & =\left[\Delta p_{1 r, j}^{1_{1} 2_{1}}, \ldots, \Delta p_{1 r, j}^{1_{1} m_{1}}, \Delta p_{1 r, j}^{1_{1} 1_{2}}, \ldots, \Delta p_{1 r, j}^{1_{1} m_{2}}, \ldots, \Delta p_{1 r, j}^{1_{1} 1_{\eta}}, \ldots, \Delta p_{1 r, j}^{1_{1} m_{\eta}}\right]^{T} \\
y_{\phi ; j} & =\left[\Delta \phi_{1 r, j}^{1_{1} 2_{1}}, \ldots, \Delta \phi_{1 r, j}^{1_{1} m_{1}}, \Delta \phi_{1 r, j}^{1_{1} 1_{2}}, \ldots, \Delta \phi_{1 r, j}^{1_{1} m_{2}}, \ldots \Delta \phi_{1 r, j}^{1_{1} 1_{\eta}}, \ldots, \Delta \phi_{1 r, j}^{1_{1} m_{\eta}}\right]^{T} \\
G_{r} & =\left[g_{r}^{1_{1} 2_{1}}, \ldots, g_{r}^{1_{1} m_{1}}, g_{r}^{1_{1} 1_{2}}, \ldots, g_{r}^{1_{1} m_{2}}, \ldots, g_{r}^{1_{1} 1_{\eta}}, \ldots, g_{r}^{1_{1} m_{\eta}}\right]^{T} \\
z_{j} & =\left[N_{1 r, j}^{1_{1} 2_{1}}, \ldots, N_{1 r, j}^{1_{1} m_{1}}, N_{1 r, j}^{1_{1} 1_{2}}, \ldots, N_{1 r, j}^{1_{1} m_{2}}, \ldots N_{1 r, j}^{1_{1} 1_{\eta}}, \ldots, N_{1 r, j}^{1_{1} m_{\eta}}\right]^{T} \\
h_{p ; j} & =\left[d_{1 r, j}^{12}, \ldots, d_{1 r, j}^{1 \eta}\right]^{T} \\
h_{\phi ; j} & =\left[\delta_{1 r, j}^{12}, \ldots, \delta_{1 r, j}^{, 1 \eta}\right]^{T}
\end{aligned}
$$

and the $(m-1) \times(\eta-1)$ matrix:

$$
H_{\eta}=\left[\begin{array}{cccc}
0 & 0 & \ldots & 0 \\
e_{m_{2}} & 0 & \ldots & 0 \\
0 & e_{m_{3}} & \ldots & 0 \\
\vdots & \vdots & \ddots & \vdots \\
0 & 0 & \ldots & e_{m_{\eta}}
\end{array}\right]
$$

where $e_{n}$ is the $n \times 1$ vector of 1 's.

When combining the single-epoch, multi-frequency linearized DD GNSS code and phase observation Equations (9) and (10), we obtain the mixed integer model of observation equations:

$$
\mathrm{E}(y)=A z+G b+H h \quad z \in \mathbb{Z}^{f(m-1)}, b \in \mathbb{R}^{3}, h \in \mathbb{R}^{2 f(\eta-1)}
$$

where $y=\left[y_{\phi}^{T}, y_{p}^{T}\right]^{T}$ is the $2 f(m-1) \times 1$ vector of linearized (observed-minus-computed) DD observations, with $y_{\phi}=\left[y_{\phi ; 1}{ }^{T}, \ldots, y_{\phi ; f^{T}}\right]^{T}, y_{p}=\left[y_{p ; 1}{ }^{T}, \ldots, y_{p ; f^{T}}\right]^{T}$, and $z=\left[z, 1^{T}, \ldots, z_{, f^{T}}\right]^{T}$ is the $f(m-1) \times 1$ vector of unknown DD integer ambiguities, $h=\left[h_{\phi}^{T}, h_{p}^{T}\right]^{T}$ is the $2 f(\eta-1) \times 1$ vector of ISTBs with $h_{\phi}=\left[h_{\phi ; 1}{ }^{T}, \ldots, h_{\phi ; f}{ }^{T}\right]^{T}$ and $h_{p}=\left[h_{p ; 1}{ }^{T}, \ldots, h_{p ; f^{T}}\right]^{T}, G=e_{2} \otimes e_{f} \otimes G_{r}$ is the $2 f(m-1) \times 3$ geometry matrix, $A=\left[L^{T}, 0^{T}\right]^{T}$ is the $2 f(m-1) \times f(m-1)$ design matrix with $f(m-1) \times f(m-1)$ matrix $L=\operatorname{diag}\left(\lambda_{1}, \ldots, \lambda_{f}\right) \otimes I_{m-1}, H=\left[H_{\phi}^{T}, H_{p}^{T}\right]$ is the $2 f(m-1) \times f(\eta-1)$ design matrix with $f(m-1) \times f(\eta-1)$ matrix $H_{\phi}=\operatorname{diag}\left(\lambda_{1}, \ldots, \lambda_{f}\right) \otimes H_{\eta}$ and $f(m-1) \times f(\eta-1)$ matrix $H_{p}=I_{f} \otimes H_{\eta}$, diag refers to the diagonal matrix formed by given arguments and $\otimes$ denotes the Kronecker product [51,52]. The model in Equation (18) contains a rank deficiency of $f(\eta-1)$, and it is not possible to simultaneously estimate the differential phase ISTB parameters and the double difference ambiguities of satellites that do not belong to the type of the pivot satellite. In the following, we describe four different processing strategies, namely ignoring ISTBs, removing rank deficiency, eliminating ISTBs and correcting for ISTBs. 


\subsection{Classical DD Model}

The naive approach is to simply ignore the third term in Equation (18), resulting in the classical baseline model with a full-rank system:

$$
\mathrm{E}(y)=A z+G b \quad z \in \mathbb{Z}^{f(m-1)}, b \in \mathbb{R}^{3}
$$

Hence, the redundancy of this classical model is equal to $2 f(m-1)-f(m-1)-3=f(m-1)-3$. As demonstrated in Section 4.3, ignoring the ISTBs results in catastrophic failure of ambiguity resolution.

\subsection{Extended DD Model with Estimable ISTBs}

The rank deficiency in Equation (18) can be removed by constraining a set of parameters (combinations) as the S-basis [53-55]. The number of S-basis constraints equals the size of the rank deficiency. There are many possibilities to choose S-basis constraints. One such choice corresponds to a reparametrization in Equation (6), such that the DD ambiguities of the pivot satellites of second satellite types are combined with corresponding phase ISTBs:

$$
\begin{aligned}
& \phi_{1 r, j}^{1_{1} 1_{\beta}}=\rho_{1 r}^{1_{1} 1_{\beta}}+\lambda_{j} \bar{\delta}_{1 r, j}^{, 1 \beta}+\epsilon_{1 r, j}^{1_{1} 1_{\beta}}, \quad \text { for } \beta \neq 1 \\
& \phi_{1 r, j}^{1_{1} s_{\beta}}=\rho_{1 r}^{1_{1} s_{\beta}}+\lambda_{j}\left(\bar{\delta}_{1 r, j}^{, 1 \beta}+N_{1 r, j}^{1_{\beta} s_{\beta}}\right)+\epsilon_{1 r, j}^{1_{1} s_{\beta}} \quad \text { for } \beta \neq 1, s_{\beta} \neq 1
\end{aligned}
$$

where $1_{\beta}$ is the pivot satellite of type- $\beta$ satellites, $\bar{\delta}_{1 r, j}^{1 \beta}=N_{1 r, j}^{1_{1} 1_{\beta}}+\delta_{1 r, j}^{, 1 \beta}$ and $N_{1 r, j}^{1_{\beta} s_{\beta}}=N_{1 r, j}^{1_{1} s_{\beta}}-N_{1 r, j}^{1_{1} 1_{\beta}}$. The estimable phase ISTB, $\bar{\delta}_{1 r, j}^{, 1 \beta}$, is now biased by the inter-satellite-type ambiguity between the pivot satellites. Hence, to avoid the jumps due to the changes of reference satellites and cycle slips, we report the fractional part of the estimable phase ISTBs, which are sufficient for ISTB correction, as discussed in Section 2.4. Instead of lumping phase ISTB with the ambiguity of the first (pivot) satellite, one can lump the phase ISTB with the DD ambiguity of the satellite, other than the first satellite of second satellite type, and end up with different S-bases and different estimable phase ISTBs.

Another option, i.e., another S-basis choice, is to lump the phase ISTB with the average of two or more DD ambiguities of the second satellite type. For example, if we lump the phase ISTB with the average of all DD ambiguities of the second satellite type, the DD phase observations of the second satellite type read:

$$
\begin{aligned}
& \phi_{1 r, j}^{1_{1} 1_{\beta}}=\rho_{1 r}^{1_{1} 1_{\beta}}-\frac{\lambda_{j}}{m_{\beta}} \sum_{s_{\beta}=2}^{m_{\beta}} N_{1 r, j}^{\overline{1}_{\beta} s_{\beta}}+\lambda_{j} \overline{\bar{\delta}}_{1 r, j}^{1 \beta}+\epsilon_{1 r, j}^{1_{1} 1_{\beta}}, \quad \text { for } \beta \neq 1 \\
& \phi_{1 r, j}^{1_{1} s_{\beta}}=\rho_{1 r}^{1_{1} s_{\beta}}+\frac{\lambda_{j}}{m_{\beta}} N_{1 r, j}^{\overline{1}_{\beta} s_{\beta}}+\lambda_{j} \overline{\bar{\delta}}_{1 r, j}^{1 \beta}+\epsilon_{1 r, j}^{1_{1} s_{\beta}} \quad \text { for } \beta \neq 1, s_{\beta} \neq 1
\end{aligned}
$$

with estimable integer ambiguities:

$$
N_{1 r, j}^{\overline{1}_{\beta} s_{\beta}}=\sum_{i_{\beta}=1}^{m_{\beta}}\left(N_{1 r, j}^{1_{1} s_{\beta}}-N_{1 r, j}^{1_{1} i_{\beta}}\right), \quad s_{\beta}=2, \ldots, m_{\beta}
$$


and estimable phase ISTB:

$$
\overline{\bar{\delta}}_{1 r, j}^{1 \beta}=\delta_{1 r, j}^{, 1 \beta}+\frac{1}{m_{\beta}} \sum_{s_{\beta}=1}^{m_{\beta}} N_{1 r, j}^{1_{1} s_{\beta}}
$$

For this choice of S-basis, however, the fractional part of the estimable phase ISTB is not necessarily equal to that of the actual phase ISTB. Having different choices, one should be cautious while interpreting or using the estimated phase ISTBs based on a given S-basis choice.

In this contribution, we consider the parametrization of Equations (20) and (21), which has the simple interpretation that the estimable integer ambiguities correspond to satellite type-specific double differencing (see Section 2.3), and moreover, it enables us to observe the fractional parts of the phase ISTBs (see Section 4.2). For our choice of S-basis, the full-rank (extended) model reads:

$$
\mathrm{E}(y)=\bar{A} \bar{z}+G b+H \bar{h} \quad \bar{z} \in \mathbb{Z}^{f(m-\eta)}, b \in \mathbb{R}^{3}, \bar{h} \in \mathbb{R}^{2 f(\eta-1)}
$$

where $\bar{A}=\left[\bar{L}^{T}, 0^{T}\right]^{T}$ is the $2 f(m-1) \times f(m-\eta)$ design matrix with $f(m-1) \times f(m-\eta)$ matrix $\bar{L}=\operatorname{diag}\left(\lambda_{1}, \ldots, \lambda_{f}\right) \otimes \operatorname{blockdiag}\left(I_{m_{1}-1}, C_{m_{2}}, \ldots, C_{m_{\eta}}\right), C_{n}=\left[\begin{array}{ll}0 & I_{n-1}\end{array}\right]^{T}$ and blockdiag referring to the block diagonal matrix formed by given arguments, $\bar{z}=\left[\bar{z}_{, 1}^{T}, \ldots, \bar{z}_{, f}^{T}\right]^{T}$ is the $f(m-\eta) \times 1$ vector of unknown DD integer ambiguities with $\bar{z}_{, j}=\left[\bar{z}_{, j}^{, 1_{T}}, \ldots, \bar{z}_{, j}^{, \eta_{T}}\right]^{T}$ and $\bar{z}_{, j}^{, \beta}=\left[N_{1 r, j}^{1_{\beta} 2_{\beta}}, \ldots, N_{1 r, j}^{1_{\beta} m_{\beta}}\right]^{T}$, $\bar{h}=\left[\bar{h}_{\phi}^{T}, h_{p}^{T}\right]^{T}$ is the $2 f(\eta-1) \times 1$ vector of estimable ISTBs with $\bar{h}_{\phi}=\left[\bar{h}_{\phi ; 1}^{T}, \ldots, \bar{h}_{\phi ; f}^{T}\right]^{T}$ and $\bar{h}_{\phi ; j}=\left[\bar{\delta}_{1 r, j}^{12}, \ldots, \bar{\delta}_{1 r, j}^{, 1 \eta}\right]$. The redundancy of this model is equal to $2 f(m-1)-f(m-\eta)-2 f(\eta-1)-3=$ $f(m-\eta)-3$.

\subsection{Type-Specific DD Model}

Since ISTBs are nuisance parameters, one can eliminate $2 f(\eta-1)$ ISTBs using the differencing operator, $\overline{\mathcal{D}}^{T}=I_{2} \otimes I_{f} \otimes \operatorname{blockdiag}\left(I_{m_{1}-1}, D_{m_{2}-1}^{T}, \ldots, D_{m_{\eta}-1}^{T}\right)$, in which $D_{n}^{T}=\left[-e_{n}, I_{n}\right]$ is the differencing matrix. Pre-multiplying Equation (26) by $\overline{\mathcal{D}}^{T}$ nullifies the third term and results in a typespecific DD model, which has a reference satellite (pivot) per satellite type and reads:

$$
\mathrm{E}(\bar{y})=\overline{\bar{A}} \bar{z}+\bar{G} b \quad \bar{z} \in \mathbb{Z}^{f(m-\eta)}, b \in \mathbb{R}^{3}
$$

where $\bar{y}=\overline{\mathcal{D}}^{T} y$ is the $2 f(m-\eta) \times 1$ vector of type-specific DD observations, $\overline{\bar{A}}=\overline{\mathcal{D}}^{T} \bar{A}=\left[\overline{\bar{L}}^{T}, 0^{T}\right]^{T}$ is the $2 f(m-\eta) \times f(m-\eta)$ design matrix with $f(m-\eta) \times f(m-\eta)$ matrix $\overline{\bar{L}}=\operatorname{diag}\left(\lambda_{1}, \ldots, \lambda_{f}\right) \otimes$ blockdiag $\left(I_{m_{1}-1}, I_{m_{2}-1}, \ldots, I_{m_{\eta}-1}\right)$ and $\bar{G}=\overline{\mathcal{D}}^{T} G$ is the $2 f(m-\eta) \times 3$ geometry matrix. This model has $2 f(\eta-1)$ less observations and $2 f(\eta-1)$ less unknown parameters than Equation (26). Hence, both models have the same redundancy and are equivalent.

\subsection{ISTB-Corrected DD Model}

As shown in Section 4.2, ISTBs are stable and can be assumed to be constant for a given receiver type pair. Hence, one can correct DD observations in Equation (18) with a priori estimates of ISTBs (calibration):

$$
\mathrm{E}(\tilde{y})=A z+G b \quad z \in \mathbb{Z}^{f(m-1)}, b \in \mathbb{R}^{3}
$$


with $\tilde{y}=y-H \tilde{h}$ and $\tilde{h}$ the vector of a priori ISTBs known through calibration and consisting of code and fractional phase ISTBs (see Section 4.2). Note that it is sufficient to use the fractional part of phase ISTB to correct phase observations, as the integer part of the phase ISTB can be lumped with the corresponding integer ambiguities without affecting integer ambiguity resolution. The redundancy of an ISTB-corrected system is equal to $f(m-1)-3$. Hence, this model is stronger than the extended model in Equation (26).

Finally, Table 1 summarizes the full-rank models described in the above. For further analyses in this paper, we only consider three models, namely, the classical DD model, ignoring ISTBs, the extended model and the classical DD model with ISTB correction. The type-specific DD model is equivalent to the extended DD model.

Table 1. Redundancies of models considered. DD, double differencing; ISTB, inter-satellitetype bias.

\begin{tabular}{ll}
\hline Model & Redundancy \\
\hline Classical DD with ignoring ISTBs Equation (19) & $f(m-1)-3$ \\
Extended model (26) & $f(m-\eta)-3$ \\
Type-specific DD model (27) & $f(m-\eta)-3$ \\
Classical DD with ISTB correction Equation (28) & $f(m-1)-3$ \\
\hline
\end{tabular}

\subsection{Stochastic Model}

We assume elevation-dependent noise characteristics [56] for the undifferenced observables in Equations (1) and (2). That is, the standard deviation of the undifferenced observable, $\varsigma$, can be written as:

$$
\sigma_{\varsigma}(\theta)=\sigma_{\varsigma_{0}}\left(1+a_{\varsigma_{0}} \exp \left(\frac{-\theta}{\theta_{\varsigma_{0}}}\right)\right)
$$

where $\theta$ is the elevation angle of the corresponding satellite and $\sigma_{\varsigma_{0}}, a_{\varsigma_{0}}$ and $\theta_{\varsigma_{0}}$ are the elevation-dependent model parameters. We further assume that the receivers have similar characteristics and that the observation noise standard deviations can be decomposed as follows:

$$
\begin{array}{ccc}
\sigma_{\phi_{r, j}^{s_{\beta}}} & = & \sigma_{r} \sigma_{\phi_{0}} \sigma_{, j} \iota^{s_{\beta}} \\
\sigma_{p_{r, j}^{s_{\beta}}} & = & \sigma_{r} \sigma_{p_{0}} \sigma_{, j} \iota^{s_{\beta}} \\
\iota^{s_{\beta}} & = & \left(1+a_{0} \exp \left(\frac{-\theta^{s_{\beta}}}{\theta_{0}}\right)\right)
\end{array}
$$

where $\sigma_{r}$ and $\sigma_{, j}$ are the receiver, the frequency and the satellite-type-dependent weightings, respectively, and $\sigma_{\phi_{0}}$ and $\sigma_{p_{0}}$ are observation-dependent weightings. 
To construct the stochastic model for the observations in Equation (18), consider the undifferenced observations reading:

$$
\zeta=\left[\zeta_{1}^{T}, \zeta_{2}^{T}\right]^{T}
$$

where $\zeta_{r}=\left[\begin{array}{ll}\phi_{r}^{T}, & p_{r}^{T}\end{array}\right]^{T}, \phi_{r}=\left[\begin{array}{lll}\phi_{r, 1} & \ldots & \phi_{r, f}^{T}\end{array}\right]^{T}, \phi_{r, j}=\left[\begin{array}{ll}\phi_{r, j}^{1_{1}}, \ldots, \phi_{r, j}^{m_{1}}, \ldots \phi_{r, j}^{1_{\eta}}, \ldots, \phi_{r, j}^{m_{\eta}}\end{array}\right]^{T}, p_{r}=$ $\left[p_{r, 1}{ }^{T} \ldots p_{r, f}\right]^{T}, p_{r, j}=\left[p_{r, j}^{1_{1}}, \ldots, p_{r, j}^{m_{1}}, \ldots p_{r, j}^{1_{\eta}}, \ldots, p_{r, j}^{m_{\eta}}\right]^{T}$ and $p_{r, j}^{s_{\beta}}$ and $\phi_{r, j}^{s_{\beta}}$ are the undifferenced code and phase observations defined in Equations (1) and (2). Using the noise characteristics of Equation (30) and assuming that the observables are normally distributed and mutually uncorrelated, the dispersion matrix of the observation vector, $\zeta$, can be written as:

$$
\mathrm{D}(\zeta)=Q_{r} \otimes Q_{t} \otimes Q_{f} \otimes Q_{\theta}
$$

where $\mathrm{D}(\cdot)$ denotes the dispersion operator, $Q_{r}=\operatorname{diag}\left[\sigma_{1}^{2}, \sigma_{2}^{2}\right], Q_{t}=\operatorname{diag}\left[\sigma_{\phi_{0}}^{2}, \sigma_{p_{0}}^{2}\right]$, $Q_{f}=\operatorname{diag}\left[\begin{array}{lll}\sigma_{, 1}^{2} & \ldots & \sigma_{, f}^{2}\end{array}\right]$ are the co-factor matrices and $Q_{\theta}=\operatorname{blockdiag}\left(Q_{\theta}^{, 1}, \ldots, Q_{\theta}^{, \eta}\right)$ is the elevation-dependent weight matrix with $Q_{\theta}^{, \beta}=\operatorname{diag}\left[\left(\iota^{1_{\beta}}\right)^{2}, \ldots,\left(\iota^{m_{\beta}}\right)^{2}\right]$. Using the DD operator, $\mathcal{D}^{T}=D_{1}^{T} \otimes I_{2} \otimes I_{f} \otimes D_{m-1}^{T}$, the dispersion matrix of the DD observations is then given as:

$$
\begin{aligned}
\mathrm{D}(y)=Q_{y y} & =\mathrm{D}\left(\mathcal{D}^{T} \zeta\right) \\
& =\left(\sigma_{1}^{2}+\sigma_{2}^{2}\right) Q_{t} \otimes Q_{f} \otimes\left(D_{m-1}^{T} Q_{\theta} D_{m-1}\right)
\end{aligned}
$$

For type-specific DD observations in Equation (27), the dispersion matrix is given as:

$$
\begin{aligned}
\mathrm{D}(\bar{y})=Q_{\bar{y} \bar{y}} & =\overline{\mathcal{D}}^{T} Q_{y y} \overline{\mathcal{D}} \\
& =\left(\sigma_{1}^{2}+\sigma_{2}^{2}\right) Q_{t} \otimes Q_{f} \otimes \operatorname{blockdiag}\left(D_{m_{1}-1}^{T} Q_{\theta}^{, 1} D_{m_{1}-1}, \ldots, D_{m_{\eta}-1}^{T} Q_{\theta}^{, \eta} D_{m_{\eta}-1}\right)
\end{aligned}
$$

\section{BeiDou Attitude Determination}

Since attitude determination uses rigidly mounted antennas, the baseline length is a priori known and can be used to strengthen the underlying model. With the inclusion of the baseline length constraint to the models in Equations (19), (26) or (28) and with the stochastic model in Equation (34), we obtain the GNSS compass model [42,47]:

$$
\begin{aligned}
\mathrm{E}(y) & =\mathcal{A} z+G b+\mathcal{H} h \quad\|b\|=l, z \in \mathbb{Z}^{\kappa}, b \in \mathbb{R}^{3} \\
\mathrm{D}(y) & =Q_{y y}
\end{aligned}
$$

where $l$ is the known baseline length, $\|\cdot\|$ denotes the unweighted norm and $\kappa$ is the number of integer ambiguities. The parameters for different models are defined as follows:

$$
\begin{array}{lllll}
\text { Classical model Equation (19): } & y=y, \quad \mathcal{A}=A, \quad z=z, & \mathcal{H}=[], & \boldsymbol{h}=[], & \kappa=f(m-1) \\
\text { Extended model Equation (26): } & y=y, \quad \mathcal{A}=\bar{A}, \quad z=\bar{z}, & \mathcal{H}=H, \quad \hbar=\bar{h}, \quad \kappa=f(m-\eta) \\
\text { ISTBcorrected classical model Equa- } & y=\tilde{y}, \quad \mathcal{A}=A, \quad z=z, \quad \mathcal{H}=[], \quad \hbar=[], & \kappa=f(m-1) \\
\text { tion (28): } & & &
\end{array}
$$

In the above, the baseline is constrained to lie on a sphere with radius $l\left(\mathbb{S}_{l}=\left\{b \in \mathbb{R}^{3} \mid\|b\|=l\right\}\right.$ ). Our objective is to solve for $b$ in a least-squares sense, thereby taking the integer constraints on $z$ and the 
quadratic constraint on vector $b$ into account. Hence, the least-squares minimization problem that will be solved reads:

$$
\min _{z \in \mathbb{Z}^{\kappa}, b \in \mathbb{S}_{l}}\|y-\mathcal{A} z-G b-\mathcal{H} \hbar\|_{Q_{y y}}^{2}
$$

with $\|\cdot\|_{Q}^{2}=(\cdot)^{T} Q^{-1}(\cdot)$. It is a quadratically-constrained (mixed) integer least-squares (QC-ILS) problem $[41,47]$, for which no closed-form solution is available. In the following sections, we describe the method for solving Equation (39).

\subsection{The Ambiguity Resolved Attitude}

We now describe the steps for computing the integer ambiguity resolved attitude angles.

\subsubsection{The Real-Valued Float Solution}

The float solution is defined as the solution of Equation (39) without the constraints. When we ignore the integer constraints on $z$ and the quadratic constraint on $b$, the float solutions, $\hat{z}, \hat{b}$ and $\hat{f}$, and their variance-covariance matrices are obtained as follows:

$$
\left[\begin{array}{ccc}
Q_{\hat{z} \hat{z}} & Q_{\hat{z} \hat{b}} & Q_{\hat{z} \hat{h}} \\
Q_{\hat{b} \hat{z}} & Q_{\hat{b} \hat{b}} & Q_{\hat{b} \hat{h}} \\
Q_{\hat{h} \hat{z}} & Q_{\hat{h} \hat{b}} & Q_{\hat{h} \hat{h}}
\end{array}\right]^{-1} \cdot\left[\begin{array}{c}
\hat{z} \\
\hat{b} \\
\hat{h}
\end{array}\right]=\left[\begin{array}{c}
\mathcal{A}^{T} \\
G^{T} \\
\mathcal{H}^{T}
\end{array}\right] Q_{y y}^{-1} y
$$

with:

$$
\left[\begin{array}{lll}
Q_{\hat{z} \hat{z}} & Q_{\hat{z} \hat{b}} & Q_{\hat{z} \hat{h}} \\
Q_{\hat{b} \hat{z}} & Q_{\hat{b} \hat{b}} & Q_{\hat{b} \hat{h}} \\
Q_{\hat{h} \hat{z}} & Q_{\hat{h} \hat{b}} & Q_{\hat{h} \hat{h}}
\end{array}\right]=\left(\left[\begin{array}{c}
\mathcal{A}^{T} \\
G^{T} \\
\mathcal{H}^{T}
\end{array}\right] Q_{y y}^{-1}\left[\begin{array}{lll}
\mathcal{A} & G & \mathcal{H}
\end{array}\right]\right)^{-1}
$$

The $z$-constrained solution of $b$ and its variance-covariance matrix can be obtained from the float solutions as follows:

$$
\begin{aligned}
\hat{b}(z) & =\hat{b}-Q_{\hat{b} \hat{z}} Q_{\hat{z} \hat{z}}^{-1}(\hat{z}-z) \\
Q_{\hat{b}(z) \hat{b}(z)} & =Q_{\hat{b} \hat{b}}-Q_{\hat{b} \hat{z}} Q_{\hat{z} \hat{z}}^{-1} Q_{\hat{z} \hat{b}}
\end{aligned}
$$

The $z$ - and $b$-constrained solution of $h$ and its variance-covariance matrix can be obtained from the float solutions as follows:

$$
\begin{aligned}
\hat{h}(z, b) & =\hat{\kappa}-\left[Q_{\hat{h} \hat{z}} Q_{\hat{h} \hat{b}}\right]\left[\begin{array}{ll}
Q_{\hat{z} \hat{z}} & Q_{\hat{z} \hat{b}} \\
Q_{\hat{b} \hat{z}} & Q_{\hat{b} \hat{b}}
\end{array}\right]^{-1}\left[\begin{array}{c}
\hat{z}-z \\
\hat{b}-b
\end{array}\right] \\
Q_{\hat{h}(z, b) \hat{h}(z, b)} & =Q_{\hat{\hat{h} \hat{h}}}-\left[Q_{\hat{h} \hat{z}} Q_{\hat{h} \hat{b}}\right]\left[\begin{array}{ll}
Q_{\hat{z} \hat{z}} & Q_{\hat{z} \hat{b}} \\
Q_{\hat{b} \hat{z}} & Q_{\hat{b} \hat{b}}
\end{array}\right]^{-1}\left[\begin{array}{c}
Q_{\hat{z} \hat{h}} \\
Q_{\hat{b} \hat{h}}
\end{array}\right]
\end{aligned}
$$


Using the above estimates, the original problem in Equation (39) can be decomposed as:

$$
\begin{aligned}
& \min _{z \in \mathbb{Z}^{\kappa}, b \in \mathbb{S}_{l}}\|y-\mathcal{A} z-G b-\mathcal{H} h\|_{Q_{y y}}^{2} \\
& =\|\hat{e}\|_{Q_{y y}}^{2}+\min _{z \in \mathbb{Z}^{\kappa}}\left(\|\hat{z}-z\|_{Q_{\hat{z} \hat{z}}}^{2}+\min _{b \in \mathbb{S}_{l}}\left[\|\hat{b}(z)-b\|_{Q_{\hat{b}(z) \hat{b}(z)}}^{2}+\min _{\hat{h}}\|\hat{h}(z, b)-\hbar\|_{Q_{\hat{h}(z, b) \hat{h}(z, b)}}^{2}\right]\right)
\end{aligned}
$$

with $\hat{e}=y-A \hat{z}-G \hat{b}-\mathcal{H} \hat{h}$ being the vector of least-squares residuals. Note that the first term on the right-hand side of Equation (46) does not depend on the unknown parameters, $z, b$ and $f$, and is therefore constant. Unlike the third term, which is constrained, the last term can be reduced to zero for any given $z$ and $b$.

\subsubsection{The Integer Ambiguity Resolution}

Based on the orthogonal decomposition Equation (46), the quadratic-constrained integer minimization can be formulated as:

$$
\check{z}=\arg \min _{z \in \mathbb{Z}^{\kappa}} C(z)
$$

with ambiguity objective function:

$$
C(z)=\|\hat{z}-z\|_{Q_{\hat{z} \hat{z}}}^{2}+\|\hat{b}(z)-\check{b}(z)\|_{Q_{\hat{b}(z) \hat{b}(z)}^{2}}^{2}
$$

where:

$$
\check{b}(z)=\arg \min _{b \in \mathbb{S}_{l}}\|\hat{b}(z)-b\|_{Q_{\hat{b}(z) \hat{b}(z)}}^{2}
$$

The cost function, $C(z)$, is the sum of two coupled terms: the first weighs the distance from the float ambiguity vector, $\hat{z}$, to the nearest integer vector, $z$, in the metric of $Q_{\hat{z} \hat{z}}$, while the second weighs the distance from the conditional float solution, $\hat{b}(z)$, to the nearest point on the sphere, $\mathbb{S}_{l}$, in the metric of $Q_{\hat{b}(z) \hat{b}(z)}$.

Unlike with the standard LAMBDA method [29], the search space of the above minimization problem is non-ellipsoidal, due to the presence of the second term in the ambiguity objective function. Moreover, its solution requires the computation of a nonlinear-constrained least-squares problem (49) for every integer vector in the search space. In the C-LAMBDA method, this problem is mitigated through the use of easy-to-evaluate bounding functions [47]. Using these bounding functions, two strategies, namely the Expansion and the Search and Shrink strategies, were developed; see, e.g., [41,45]. These techniques avoid the computation of Equation (49) for every integer vector in the search space and compute the integer minimizer, $\check{z}$, in an efficient manner.

\subsubsection{The Ambiguity Resolved Parameter Estimation}

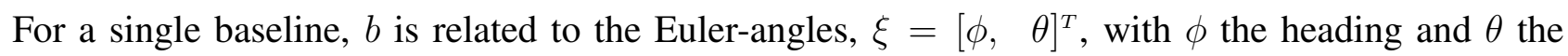
elevation, as $b(\xi)=l u(\xi)$, where $u(\xi)=\left[c_{\theta} c_{\phi}, c_{\theta} s_{\phi},-s_{\theta}\right]^{T}$, with $s_{\alpha}=\sin (\alpha)$ and $c_{\alpha}=\cos (\alpha)$. The sought for attitude angles, $\xi(\check{z})$, are the reparametrized solution of Equation (49). Using a first order 
approximation, the formal variance-covariance matrix of the ambiguity resolved, estimated heading and elevation angles are given by:

$$
Q_{\xi(\check{z}) \xi(\check{z})} \approx \frac{1}{l^{2}}\left(J_{u, \xi}(\xi(\check{z}))^{T} Q_{\hat{b}(z) \hat{b}(z)}^{-1} J_{u, \xi}(\xi(\check{z}))\right)^{-1}
$$

with Jacobian matrix:

$$
J_{u, \xi}(\xi)=\left[\begin{array}{cc}
-s_{\phi} c_{\theta} & -c_{\phi} s_{\theta} \\
c_{\phi} c_{\theta} & -s_{\phi} s_{\theta} \\
0 & -c_{\theta}
\end{array}\right]
$$

Finally, in the case of the extended model, ambiguity-corrected ISTB estimates, $\hat{\mathfrak{h}}(\check{\mathcal{z}}, \check{b}(\check{z}))$, and the associated variance-covariance matrix, $Q_{\hat{h}(z, b) \hat{h}(z, b)}$, are obtained using Equations (44) and (45). The computation of the fractional phase ISTBs from these estimable ISTBs is discussed in Section 4.2.

\section{Analyses}

\subsection{Measurement Campaign}

The analyses in this paper are based on data sets from Curtin University's permanent GNSS stations and series of zero-baseline experiments. The first data set is from Curtin's permanent GNSS antennas (CUT00 and CUTA0) mounted on the roof of building 402 at the campus of Curtin University in Perth, Australia (Figure 1a). These antennas are connected to six BeiDou-enabled receivers, as summarised in Figure 1b, consisting of three Trimble NETR9, two Javad TRE_G3T DELTA and a Septentrio POLARx4 receivers. We considered the data from these receivers for five days from April 4 to 8, 2013. BeiDou satellite visibility for April 4 is shown in Figure 2. The data of various zero baselines for five days with a $30 \mathrm{~s}$ sampling interval is used to estimate ISTBs in Section 4.2, while the data of various short baselines between CUT00 and CUTA0 on April 7 with a 1 s sampling interval is used to analyse the impact of ISTBs on attitude determination in Section 4.3.

Figure 1. Curtin Global Navigation Satellite Systems (GNSS) antennas used for the real data campaign. (a) Antenna setup; (b) antenna geometry and receiver connectivity.

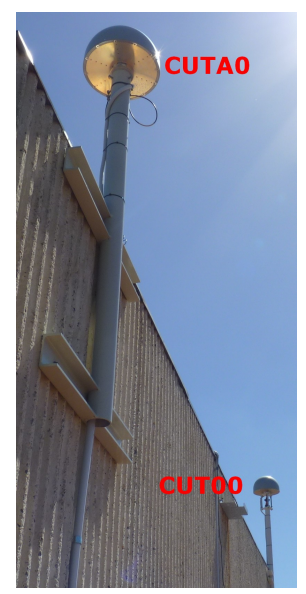

(a)

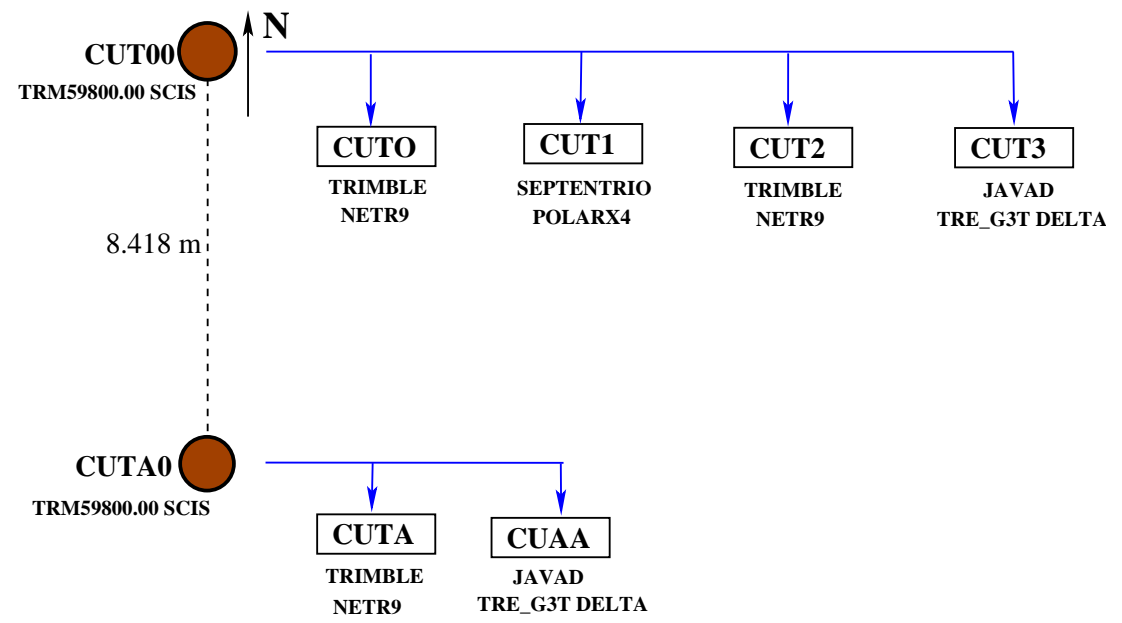

(b) 
Figure 2. Satellite visibility of BeiDou satellites for Curtin Stations on April 4, 2013 with $10^{\circ}$ elevation cut-off. (a) Skyplot; (b) number of satellites and position dilution of precision (PDOP).

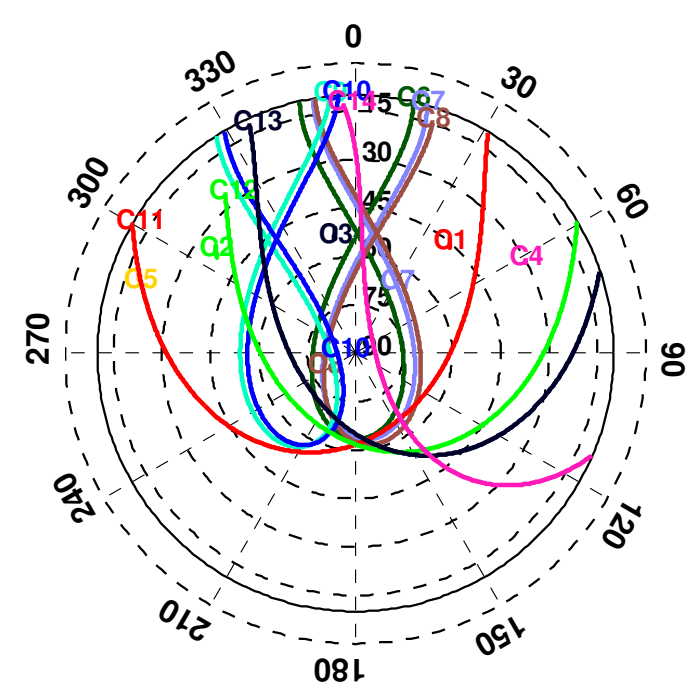

(a)

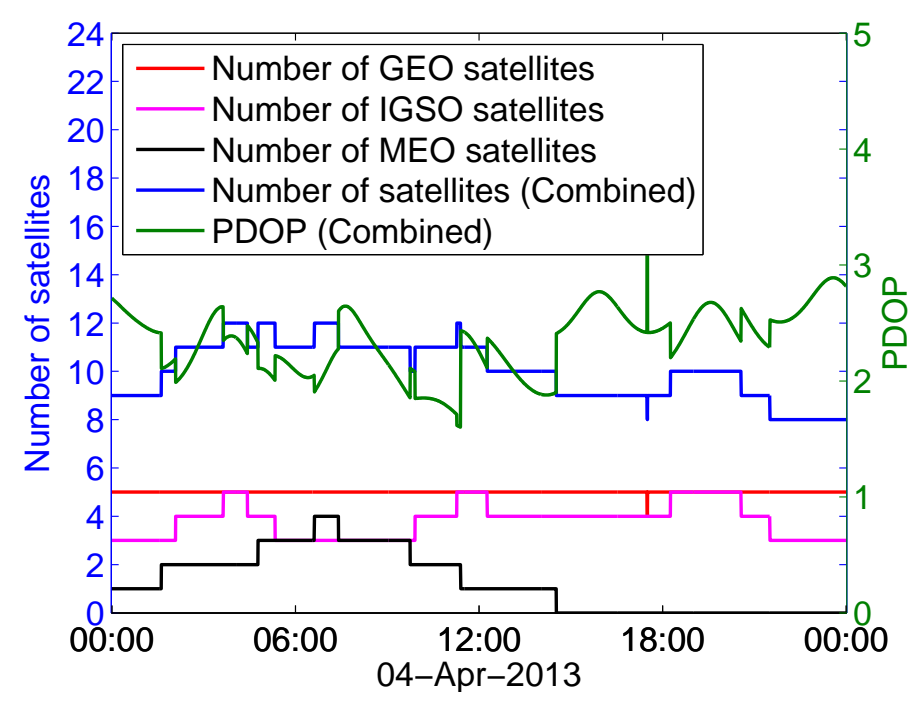

(b)

In addition to the data from Curtin's permanent stations, we also carried out a series of zero-baseline experiments: two in an open space in Curtin University and another two in an open space in Kalamunda, Western Australia (about $17 \mathrm{~km}$ from Curtin University), each for three consecutive days (Table 2). As shown in Figure 3b, a single antenna (Figure 3a) was connected to two BeiDou-enabled receivers (Trimble NETR9 and Javad Javad TRE_G3T DELTA). Figure 4 shows the visibility of BeiDou satellites on April 19, 2013. These zero-baseline data sets (with a $30 \mathrm{~s}$ sampling interval) are also used to estimate and validate ISTBs in Section 4.2. The stochastic model parameters of the elevation-dependent model Equation (29) for the receivers are reported in Table 3. Since the receivers, except Trimble NETR9, track only B1 and B2 signals, single- and dual-frequency analyses are considered in the paper.

Table 2. Zero-baseline experiments with Trimble NETR9 and Javad TRE_G3T DELTA receivers.

\begin{tabular}{ll}
\hline Experiments & Duration \\
\hline Curtin 1 & April 19-21 \\
Curtin 2 & April 29-May 01 \\
Kalamunda 1 & May 19-21 \\
Kalamunda 2 & May 29-31 \\
\hline
\end{tabular}


Figure 3. Zero-baseline experiments. (a) Antenna at Kalamunda experiment; (b) receiver-antenna connectivity.

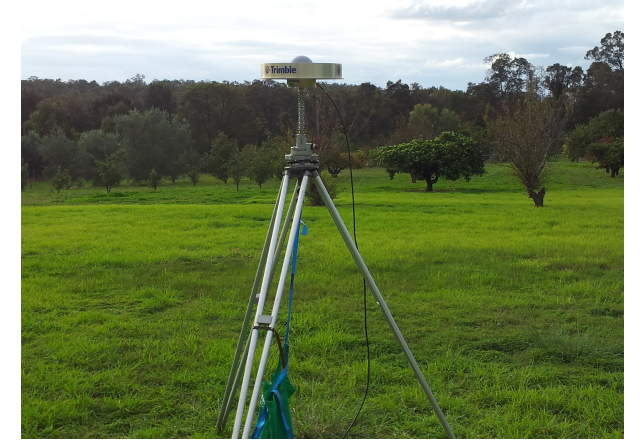

(a)

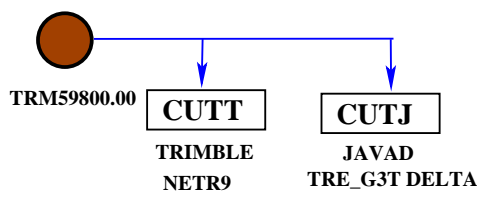

(b)

Figure 4. Satellite visibility of BeiDou satellites for open space experiment on April 19, 2013 with $10^{\circ}$ elevation cut-off. (a) Skyplot; (b) number of satellites and PDOP.

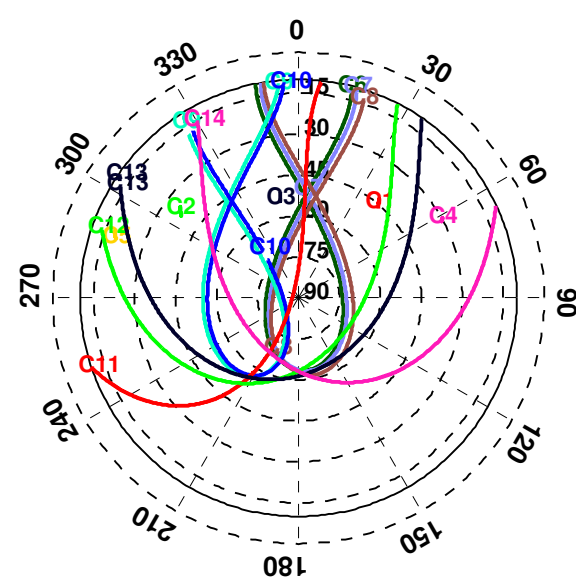

(a)

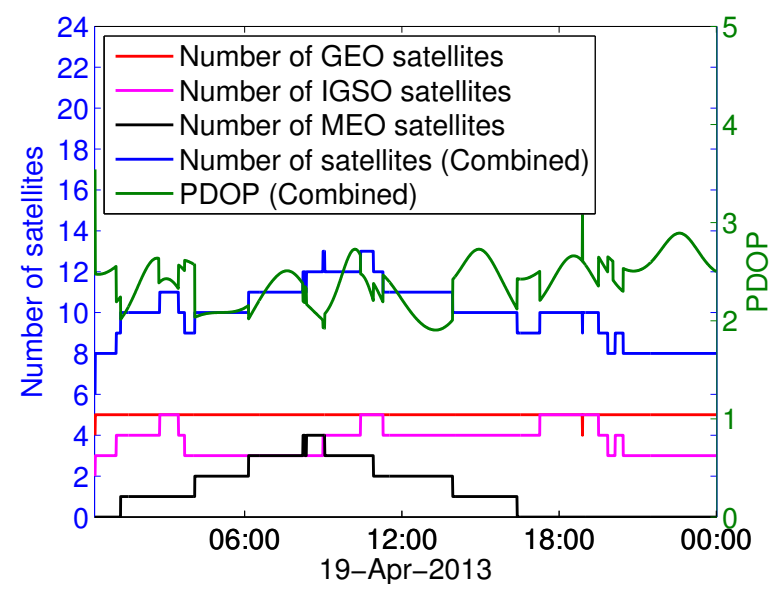

(b)

Table 3. Elevation-dependent stochastic model parameters Equation (29) used in the real data campaigns.

\begin{tabular}{lllllll}
\hline & \multicolumn{3}{c}{ Code } & \multicolumn{3}{c}{ Phase } \\
Frequency & $\begin{array}{l}\boldsymbol{\sigma}_{p_{0}} \\
{[\mathbf{c m}]}\end{array}$ & $\boldsymbol{a}_{p_{\mathbf{0}}}$ & $\boldsymbol{\epsilon}_{p_{\mathbf{0}}}$ & $\boldsymbol{\sigma}_{\phi_{0}}$ & $\boldsymbol{a}_{\phi_{\mathbf{0}}}$ & $\epsilon_{\phi_{0}}$ \\
{$[\mathbf{d e g}]$} & {$[\mathbf{m m}]$} & {$[\mathbf{d e g}]$} \\
\hline B1 and B2 & 20 & 5 & 15 & 2 & 5 & 15 \\
\hline
\end{tabular}

\subsection{BeiDou Inter-Satellite-Type Bias (ISTB)}

First, we considered the estimation of differential ISTBs using zero baseline data. Since the geometry term vanishes for a zero baseline problem, the estimation of code and phase ISTBs for each frequency 
are decoupled. Using the extended model in Equation (26) and the associated stochastic model in Equation (34), the decoupled system for the differential code ISTBs at the $j$ th frequency is given as:

$$
\begin{aligned}
\mathrm{E}\left(y_{p ; j}\right) & =H_{\eta} h_{p ; j}, \quad h_{p ; j} \in \mathbb{R}^{\eta-1} \\
\mathrm{D}\left(y_{p ; j}\right) & =Q_{y_{p ; j} y_{p ; j}}=\left(\sigma_{1}^{2}+\sigma_{r}^{2}\right) \sigma_{p_{0}}^{2} \sigma_{, j}^{2}\left(D_{m-1}^{T} Q_{\theta} D_{m-1}\right)
\end{aligned}
$$

The estimates of differential code ISTBs are then given by the least-squares solution of the above system. Similarly, using the extended model in Equation (26) and the associated stochastic model in Equation (34), the decoupled system for the differential estimable phase ISTBs at the $j$ th frequency is given as:

$$
\begin{aligned}
\mathrm{E}\left(y_{\phi ; j}\right) & =\bar{L}_{, j} \bar{z}_{, j}+\lambda_{j} H_{\eta} \bar{h}_{\phi ; j}, \quad \bar{z}_{, j} \in \mathbb{Z}^{m-\eta}, \bar{h}_{\phi ; j} \in \mathbb{R}^{\eta-1} \\
\mathrm{D}\left(y_{\phi ; j}\right) & =Q_{y_{\phi ;} y_{\phi ; j}}=\left(\sigma_{1}^{2}+\sigma_{r}^{2}\right) \sigma_{\phi_{0}}^{2} \sigma_{, j}^{2}\left(D_{m-1}^{T} Q_{\theta} D_{m-1}\right)
\end{aligned}
$$

with $\bar{L}_{, j}=\lambda_{j}$ blockdiag $\left(I_{m_{1}-1}, C_{m_{2}}, \ldots, C_{m_{\eta}}\right)$. First, the float solution of the above full-rank square system is obtained by ignoring integer constraints:

$$
\begin{aligned}
{\left[\begin{array}{c}
\hat{\bar{z}}_{, j} \\
\hat{\bar{h}}_{\phi ; j}
\end{array}\right] } & =\left[\bar{L}_{, j}, \lambda_{j} H_{\eta}\right]^{-1} y_{\phi ; j} \\
{\left[\begin{array}{cc}
Q_{\hat{\bar{z}}_{, j} \hat{\bar{z}}_{j}} & Q_{\hat{\bar{z}}_{, j} \hat{\bar{h}}_{\phi ; j}} \\
Q_{\hat{\bar{h}}_{\phi ; j} \hat{\bar{z}}_{, j}} & Q_{\hat{\bar{h}}_{\phi ; j} \hat{\bar{h}}_{\phi ; j}}
\end{array}\right] } & =\left[\bar{L}_{, j}, \lambda_{j} H_{\eta}\right]^{-1} Q_{y_{\phi ; j} y_{\phi ; j}}\left[\begin{array}{c}
\bar{L}_{, j} \\
\lambda_{j} H_{\eta}
\end{array}\right]^{-1}
\end{aligned}
$$

Since the above system is driven by phase measurement noise, simple rounding of $\hat{\bar{z}}_{, j}$ yields integer ambiguities, $\check{\bar{z}}_{, j}$. The estimates for the estimable phase ISTBs are then given by:

$$
\begin{aligned}
& \hat{\bar{h}}_{\phi ; j}\left(\check{\bar{z}}_{, j}\right)=\hat{\bar{h}}_{\phi ; j}-Q_{\hat{\bar{h}}_{\phi ; j} \hat{\bar{z}}_{j}} Q_{\hat{\bar{z}}_{, j} \hat{\bar{z}}_{, j}}^{-1}\left(\hat{\bar{z}}_{, j}-\check{\bar{z}}_{, j}\right) \\
& Q_{\hat{\bar{h}}_{\phi ; j}\left(\hat{\bar{z}}_{j, j}\right) \check{\bar{h}}_{\phi ; j}\left(\check{z}_{, j}\right)}=Q_{\hat{\bar{h}}_{\phi ; j} \hat{\bar{h}}_{\phi ; j}}-Q_{\hat{\bar{h}}_{\phi ; j} \hat{\bar{z}, j}} Q_{\hat{\bar{z}}_{, j} \hat{\bar{z}}_{, j}}^{-1} Q_{\hat{\bar{z}}_{, j} \hat{\bar{h}}_{\phi ; j}}
\end{aligned}
$$

Since these estimable phase ISTBs are the sum of actual phase ISTBs and corresponding ambiguities of reference satellites of the second satellite type, the estimates are affected by integer jumps, due to the cycle slips and the changes of reference satellites. Hence, we report only fractional phase ISTBs (the residuals of integer rounding) that are sufficient for ISTB correction as discussed in Section 2.4. That is, the fractional phase ISTBs, $\hat{\tilde{h}}_{\phi ; j}\left(\check{\bar{z}}_{, j}\right)=\hat{\bar{h}}_{\phi ; j}\left(\check{\bar{z}}_{, j}\right)$ - round $\left(\hat{\bar{h}}_{\phi ; j}\left(\check{\bar{z}}_{, j}\right)\right)$, where 'round' refers to the closest integer to the given estimate. However, these fractional phase ISTB estimates are ambiguous if they are equal to or close to a half cycle (e.g., for a half cycle, simple rounding will yield either $+0.5-\epsilon$ or $-0.5+\epsilon$, depending on the noise, $\epsilon$ ). For this situation, we use either "floor" (resulting in the residual for the nearest integer that is smaller than the given estimate) or "ceiling" (resulting in the residual for the nearest integer that is larger than the given estimate) with the sign convention in Table 4. Note that one is free to choose any sign convention, as long as it preserves the consistency of the double difference ambiguities when the reference receiver and/or the reference satellite type changes. 
Table 4. Sign convention for fractional phase ISTBs that are equal to or close to a half cycle, with Trimble as the reference receiver. GEO, Geostationary Earth Orbit; IGSO, Inclined Geosynchronous Satellite Orbit; MEO, Medium Earth Orbit.

\begin{tabular}{ll}
\hline Satellite type pairs & Rounding method/Sign \\
\hline GEO-IGSO & floor/+ \\
GEO-MEO & floor/+ \\
\hline IGSO-GEO & ceiling/- \\
IGSO-MEO & ceiling/- \\
\hline MEO-GEO & ceiling/+ \\
MEO-IGSO & floor/- \\
\hline
\end{tabular}

Figure 5-8 show the time series of ISTB estimates for zero-baselines formed by Curtin receivers (CUT0-CUT1, CUT0-CUT2, CUT0-CUT3 and CUTA-CUAA) on April 4, 2013. The first two columns are for ISTBs of GEO and MEO satellite types with an IGSO satellite as the reference, while the last column is for MEO ISTBs with respect to GEO satellites. Similar results for Curtin's open space experiment are given in Figure 9, matching with the results of the same receiver pair (Trimble-Javad) in Figure 7 and 8 . The gaps in the MEO-related time series are due to the unavailability of visible MEO satellites during those periods.

It is observed that the estimated ISTBs (monitored for several days) are very stable and can be used to calibrate BeiDou observations. In the following, we compute the ISTB corrections using epoch-by-epoch estimates of several days. Let us consider the time series of the $i$ th ITSB and associated standard deviations, $\left\{\hat{h}_{i: k}, \sigma_{h_{i: k}}\right\}_{k=1}^{K}$, where $K$ is the number of epochs and $\boldsymbol{h}_{i: k}$ is the estimated code or phase ISTB at time $k$. Assuming these estimates are uncorrelated over time, we formulate the following least-squares problem for the bias estimation:

$$
\begin{aligned}
\mathrm{E}\left(\hat{h}_{i}\right) & =e_{K} v_{i} \\
\mathrm{D}\left(\hat{h}_{i}\right) & =Q_{\hbar_{i} h_{i}}=\operatorname{diag}\left[\sigma_{\hbar_{i: 1}}, \ldots, \sigma_{h_{i: K}}\right]
\end{aligned}
$$

with $f_{i}=\left[f_{i: 1}, \ldots, h_{i: K}\right]^{T}$ the $K \times 1$ vector of epoch-by-epoch ISTB estimates for the $i$ th ISTB. The least-squares estimate (weighted mean) of the bias and its standard deviation are given as:

$$
\begin{aligned}
\hat{v}_{i} & =\frac{\sum_{k=1}^{K} \kappa_{i: k} / \sigma_{\kappa_{i: k}}^{2}}{\sum_{k=1}^{K} 1 / \sigma_{h_{i: k}}^{2}} \\
\sigma_{\hat{v}_{i}} & =\frac{1}{\sqrt{\sum_{k=1}^{K} 1 / \sigma_{h_{i: k}}^{2}}}
\end{aligned}
$$


Figure 5. Estimated BeiDou differential ISTB for CUT0-CUT1 on 4 April 2013 (Trimble-Septentrio, zero-baseline). (a) B1 ISTB for IGSO-GEO; (b) B1 ISTB for IGSO-MEO; (c) B1 ISTB for GEO-MEO; (d) B2 ISTB for IGSO-GEO; (e) B2 ISTB for IGSO-MEO; (f) B2 ISTB for GEO-MEO.
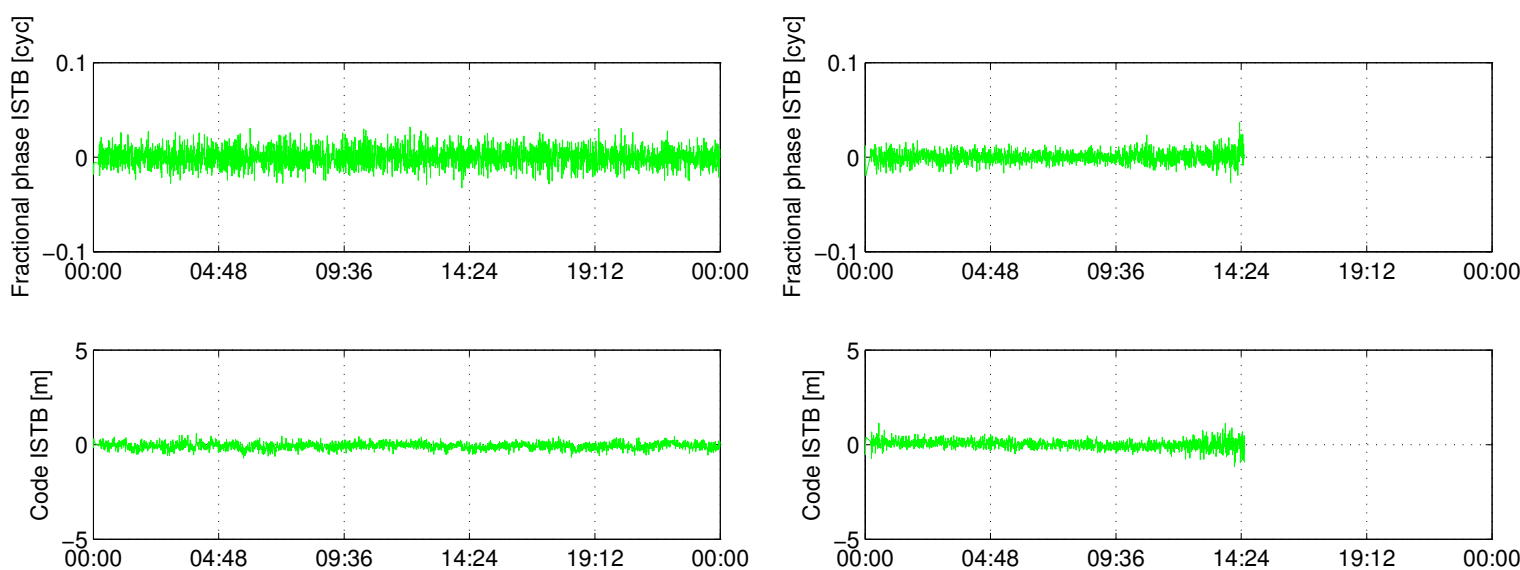

(a)

(b)
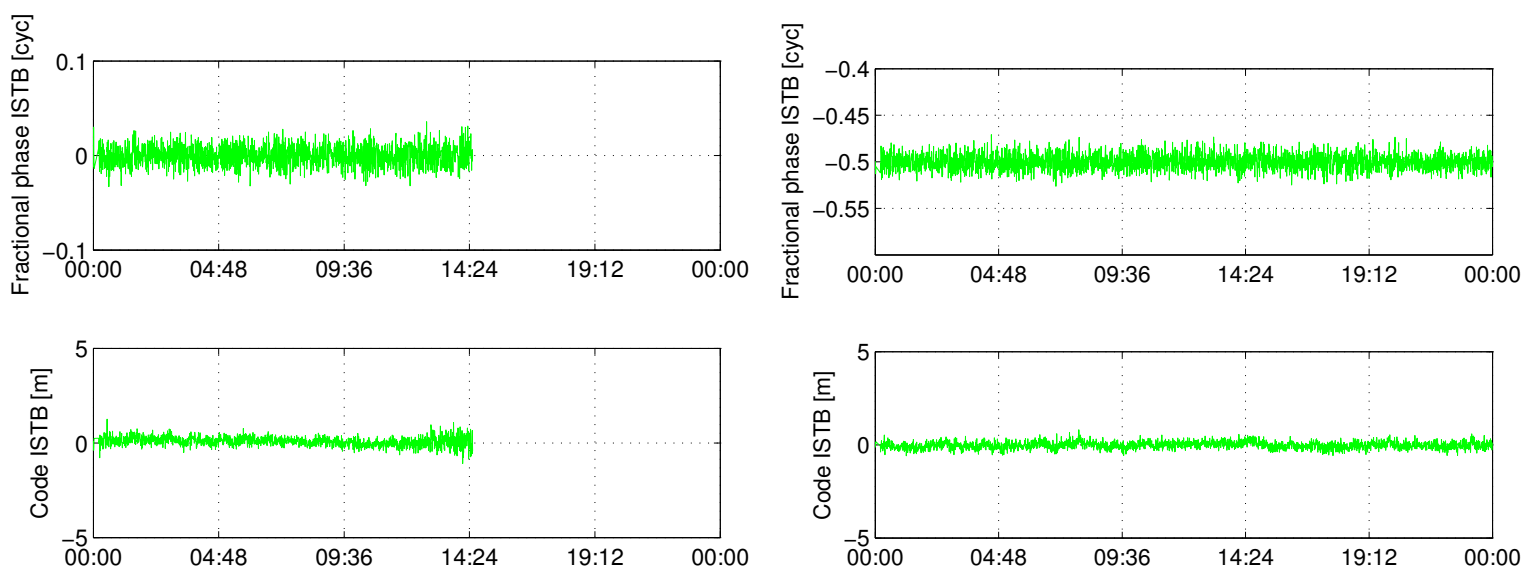

(c)

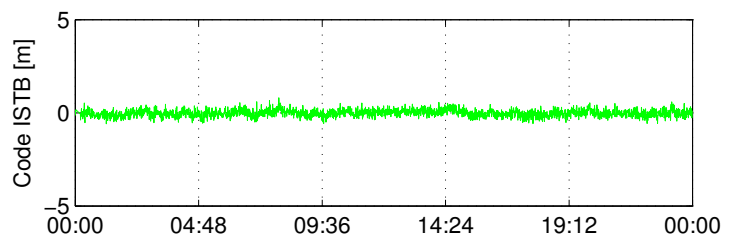

(d)
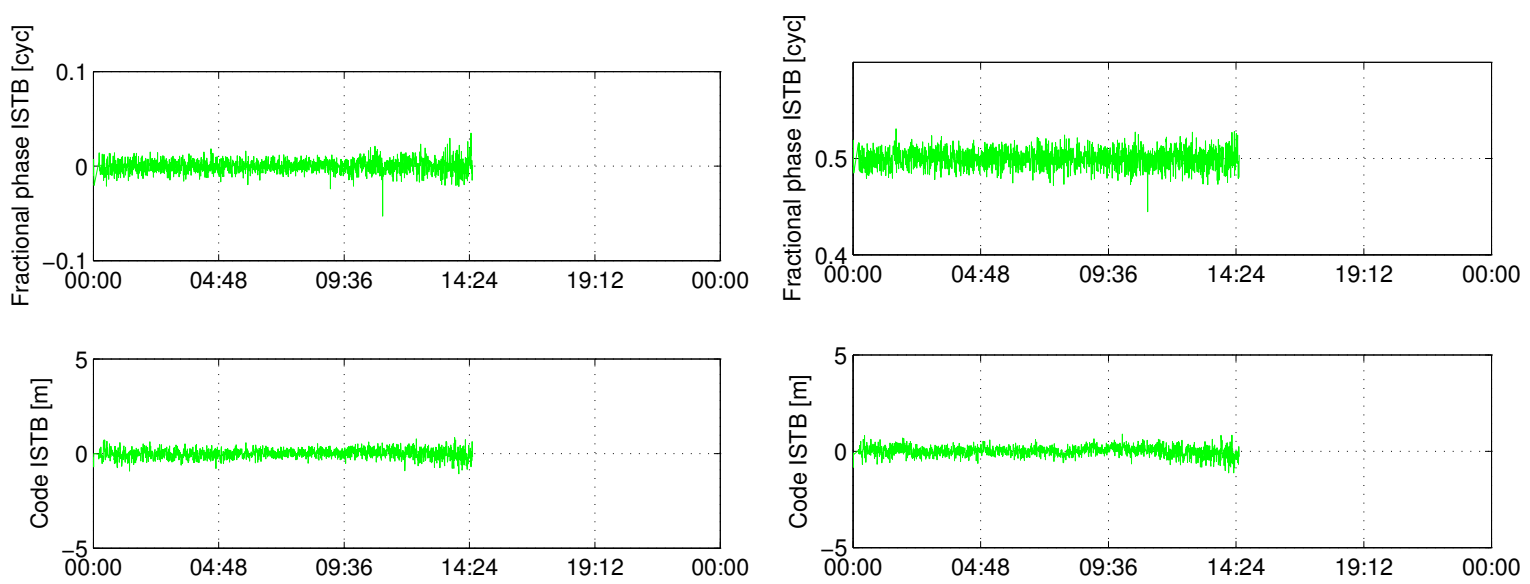

(e)

(f) 
Figure 6. Estimated BeiDou differential ISTB for CUT0-CUT2 on 4 April 2013 (Trimble-Trimble, zero-baseline). (a) B1 ISTB for IGSO-GEO; (b) B1 ISTB for IGSO-MEO; (c) B1 ISTB for GEO-MEO; (d) B2 ISTB for IGSO-GEO; (e) B2 ISTB for IGSO-MEO; (f) B2 ISTB for GEO-MEO.
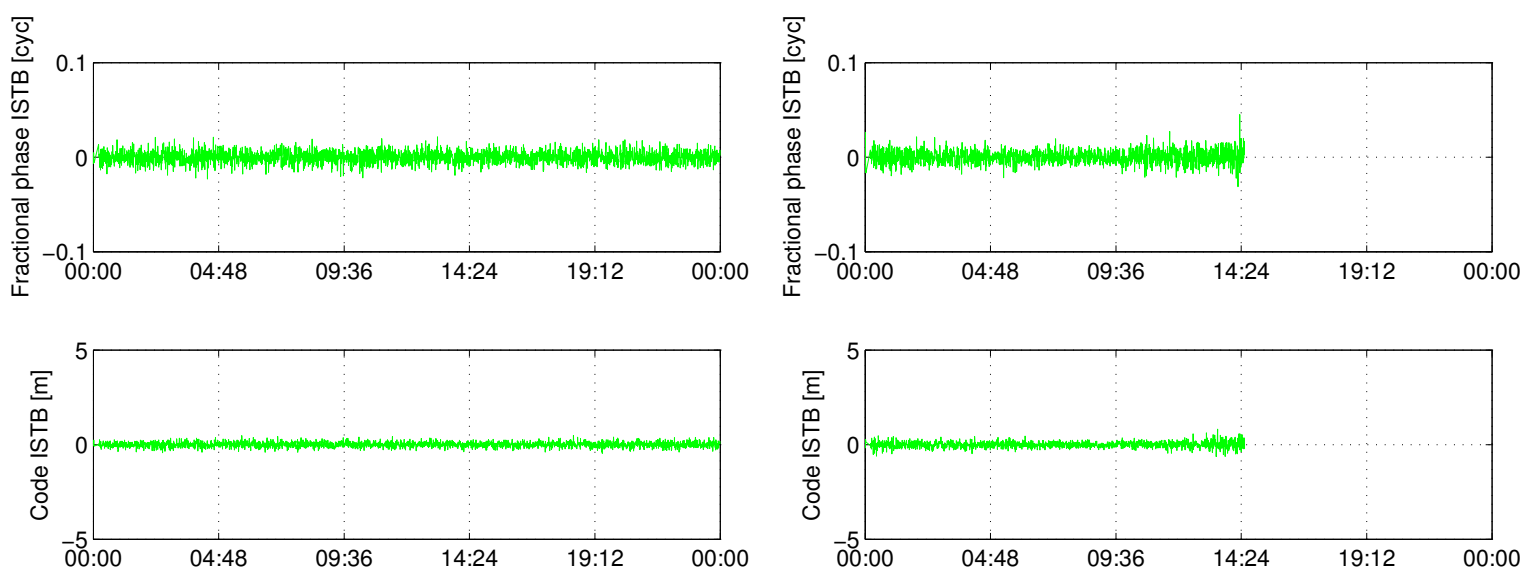

(a)

(b)
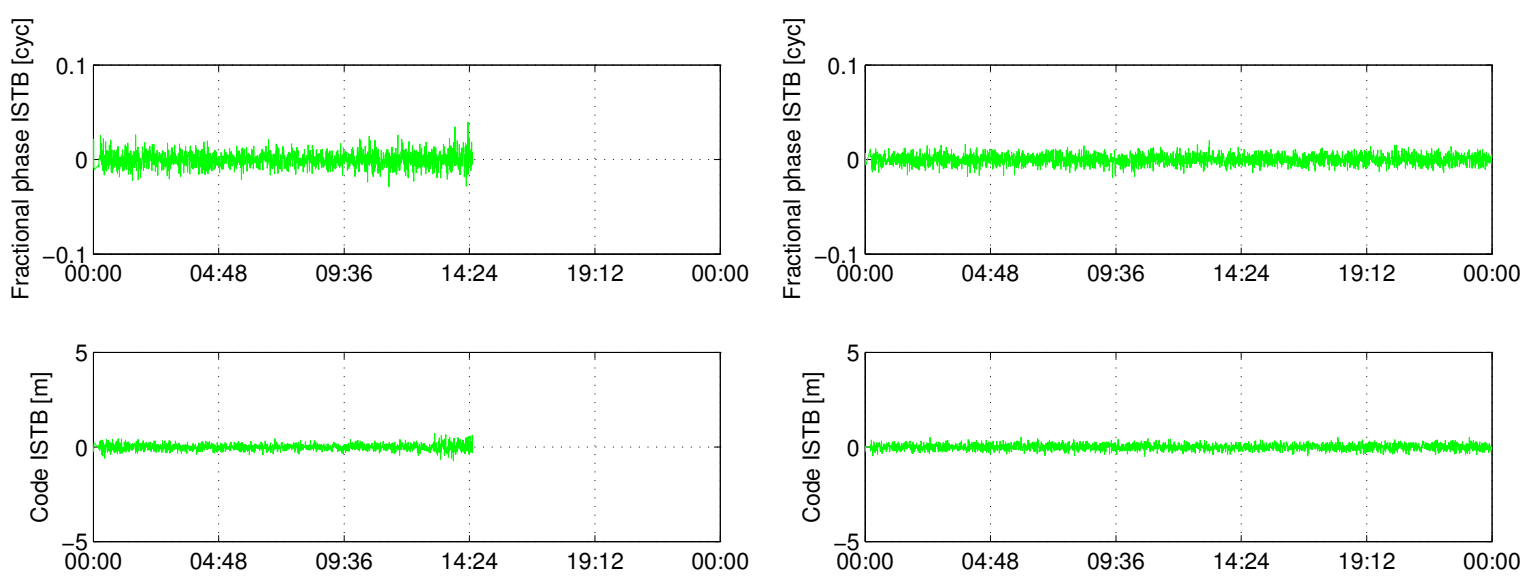

(c)

(d)
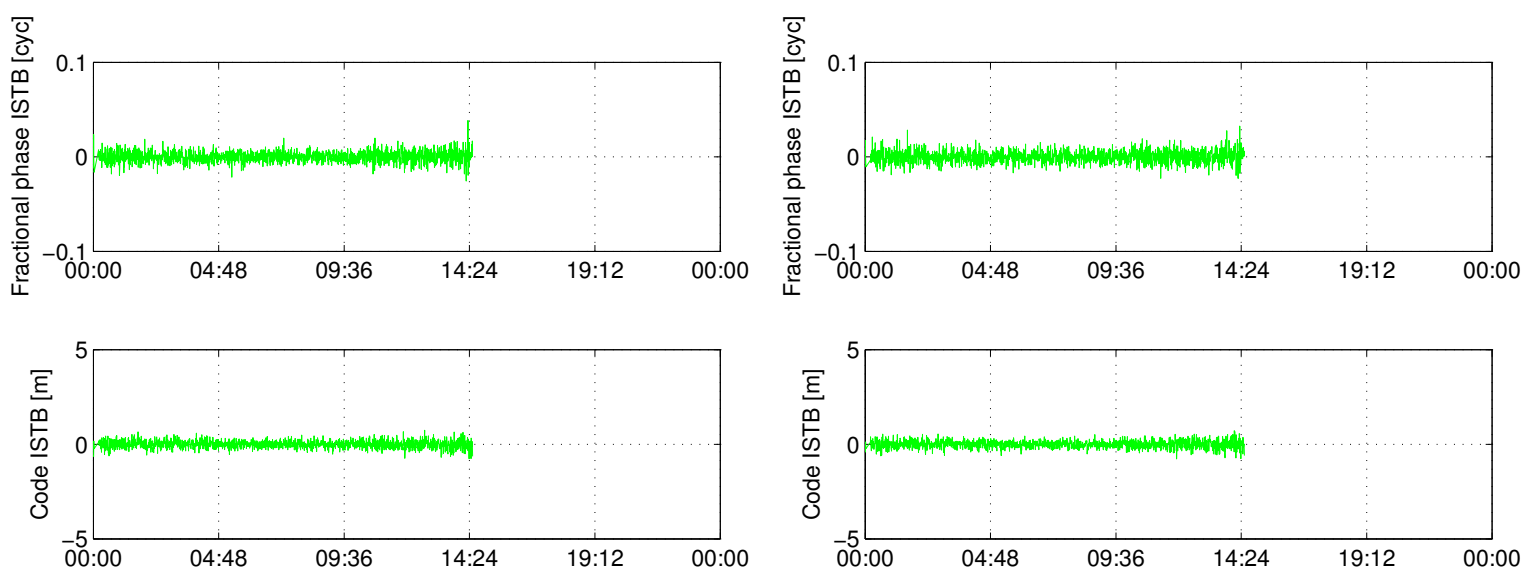

(e)

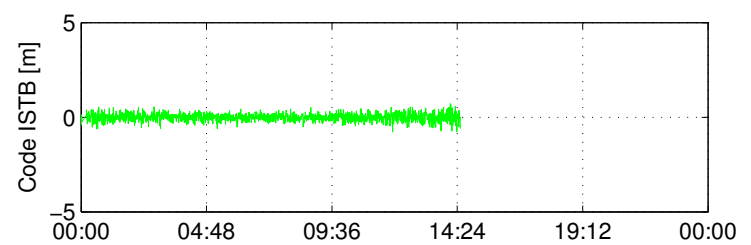

(f) 
Figure 7. Estimated BeiDou differential ISTB for CUT0-CUT3 on 4 April 2013 (Trimble-Javad, zero-baseline). (a) B1 ISTB for IGSO-GEO; (b) B1 ISTB for IGSO-MEO; (c) B1 ISTB for GEO-MEO; (d) B2 ISTB for IGSO-GEO; (e) B2 ISTB for IGSO-MEO; (f) B2 ISTB for GEO-MEO.
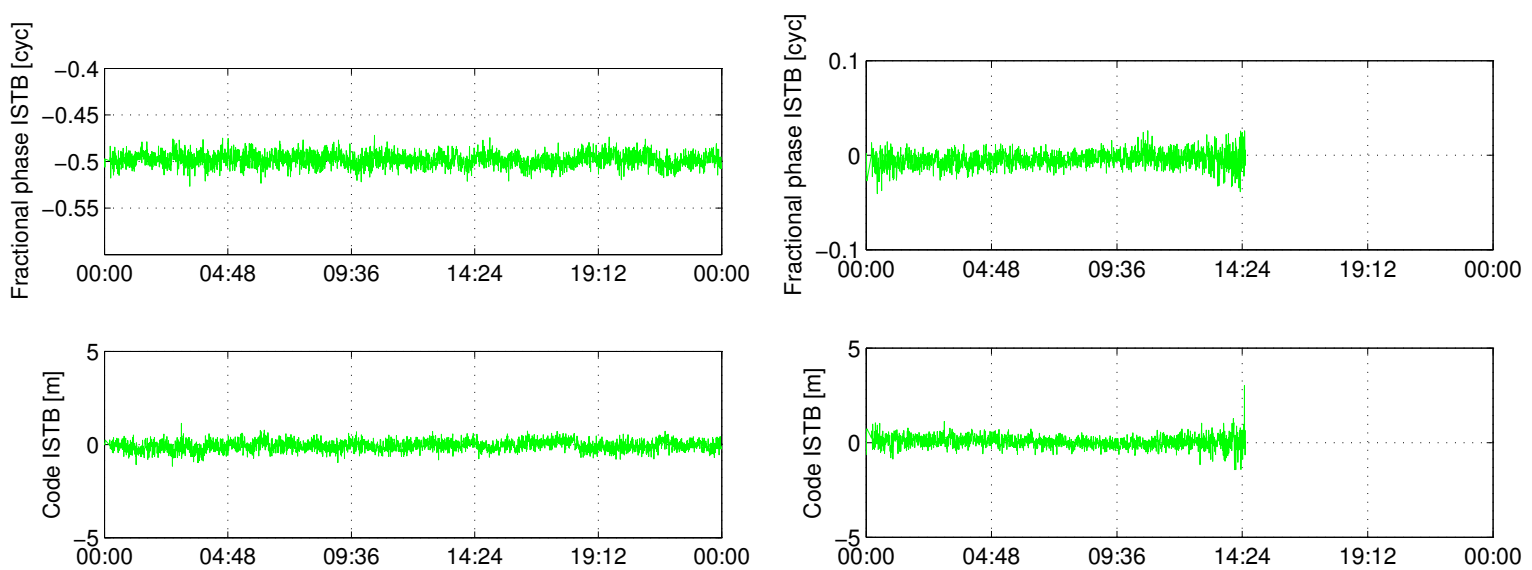

(a)

(b)
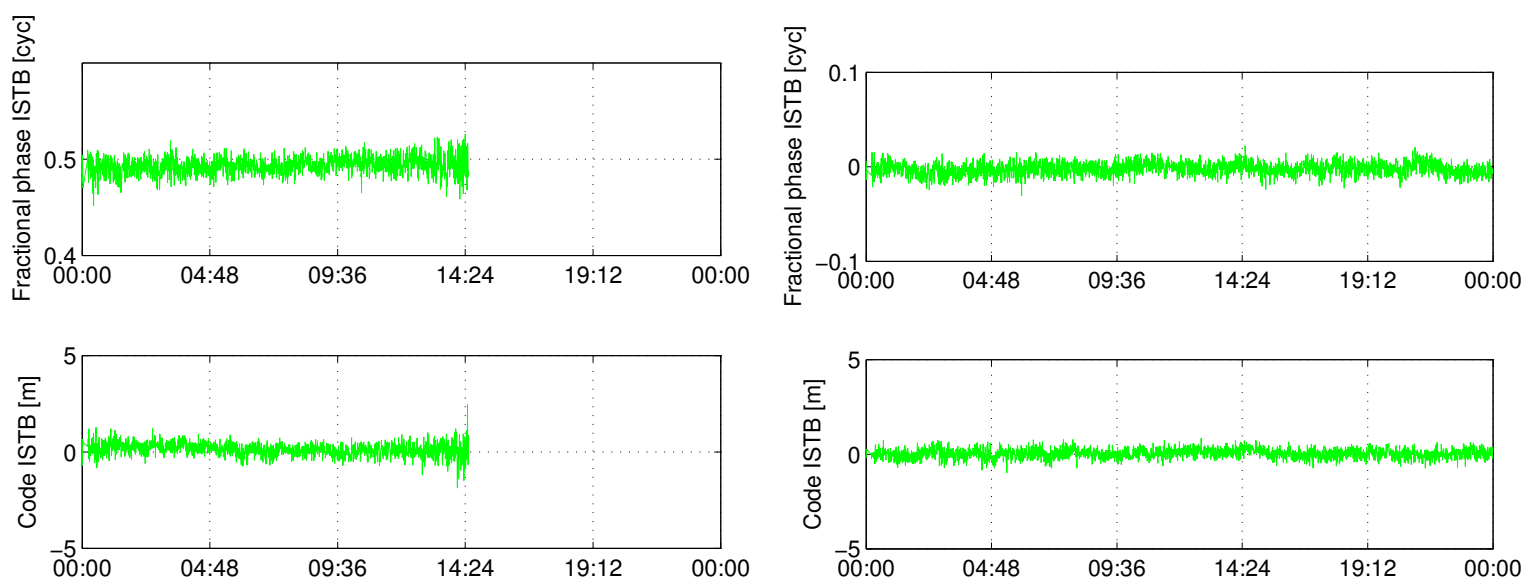

(c)

(d)
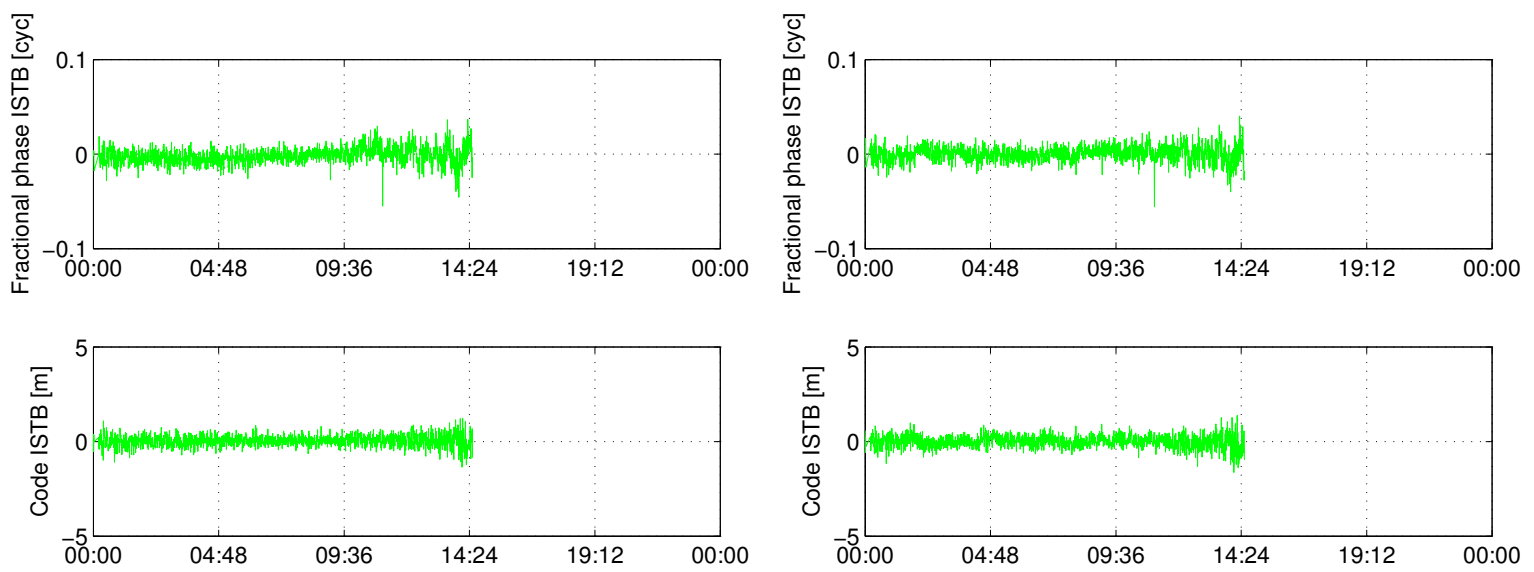

(e)

(f) 
Figure 8. Estimated BeiDou differential ISTB for CUTA-CUAA on 4 April 2013 (Trimble-Javad, zero-baseline). (a) B1 ISTB for IGSO-GEO; (b) B1 ISTB for IGSO-MEO; (c) B1 ISTB for GEO-MEO; (d) B2 ISTB for IGSO-GEO; (e) B2 ISTB for IGSO-MEO; (f) B2 ISTB for GEO-MEO.
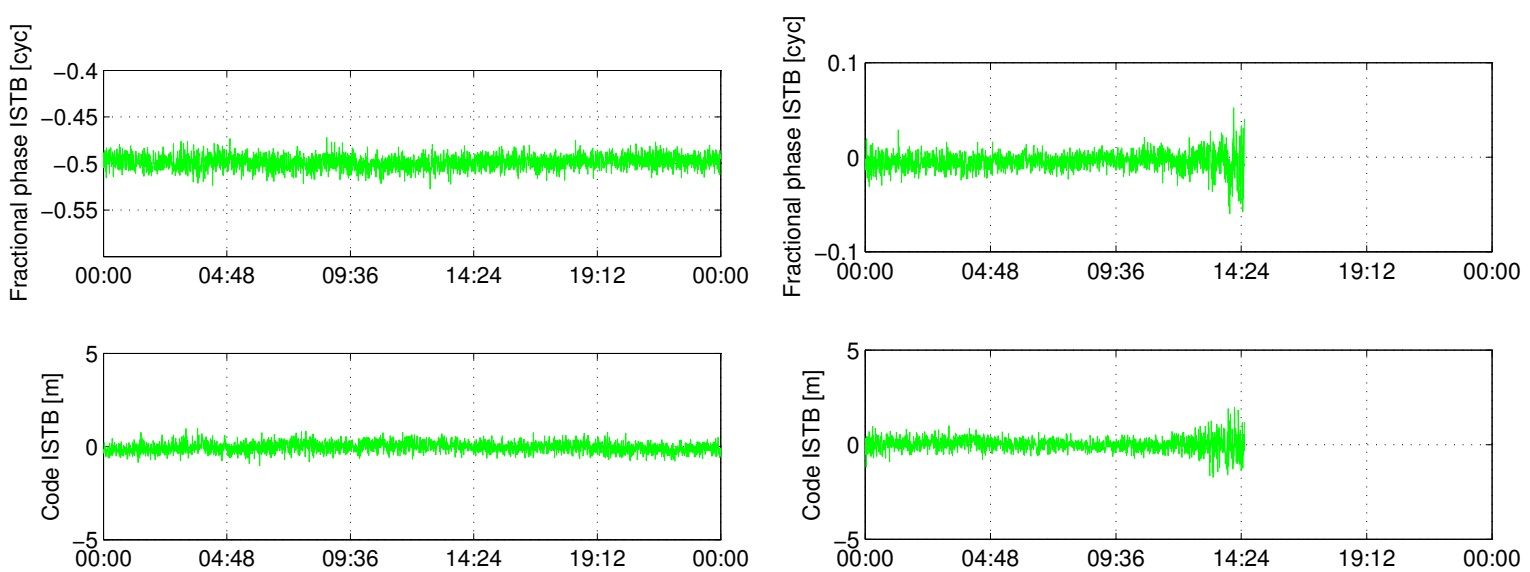

(a)

(b)
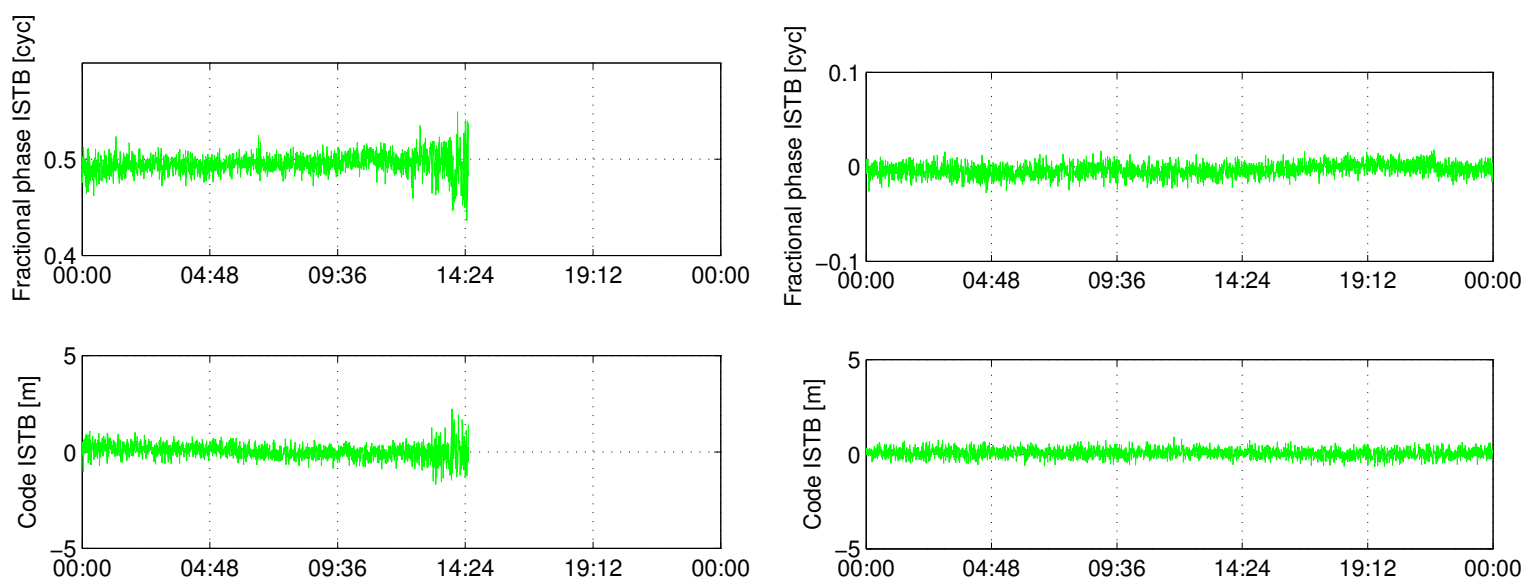

(c)

(d)
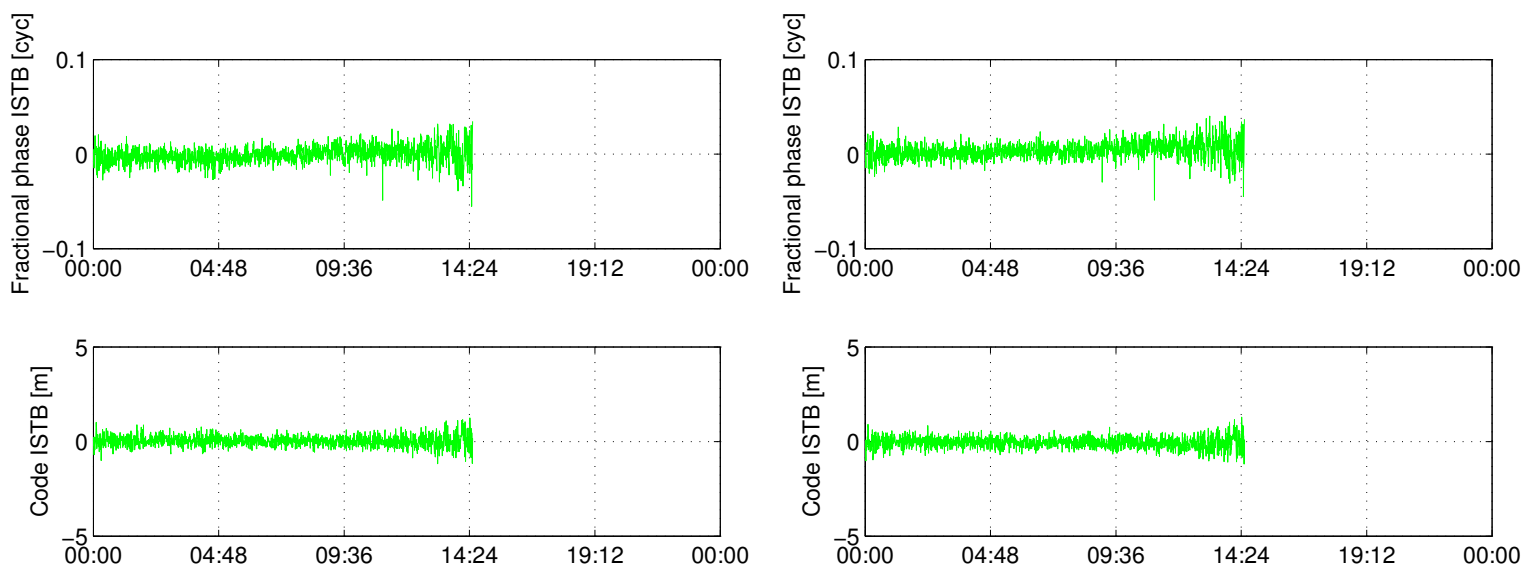

(e)

(f) 
Figure 9. Estimated BeiDou differential ISTB for CUTT-CUTJ on 19 April 2013 at Curtin open space (Trimble-Javad, zero-baseline). (a) B1 ISTB for IGSO-GEO; (b) B1 ISTB for IGSO-MEO; (c) B1 ISTB for GEO-MEO; (d) B2 ISTB for IGSO-GEO; (e) B2 ISTB for IGSO-MEO; (f) B2 ISTB for GEO-MEO.
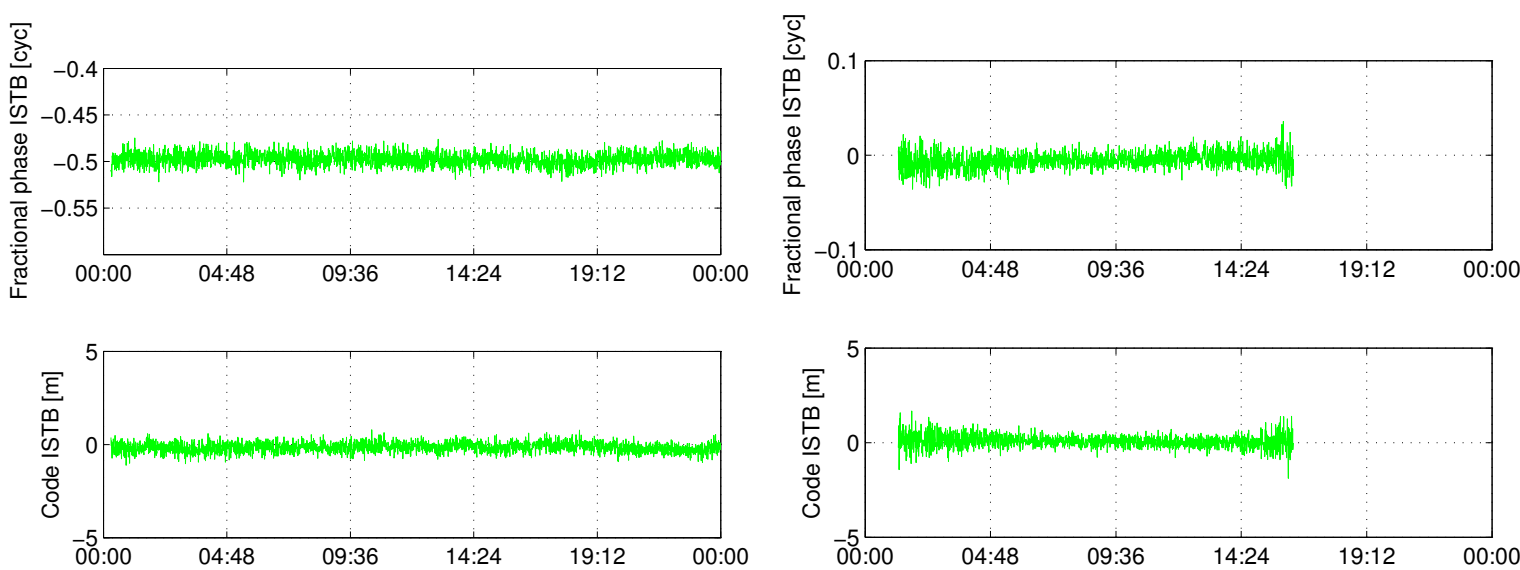

(a)

(b)
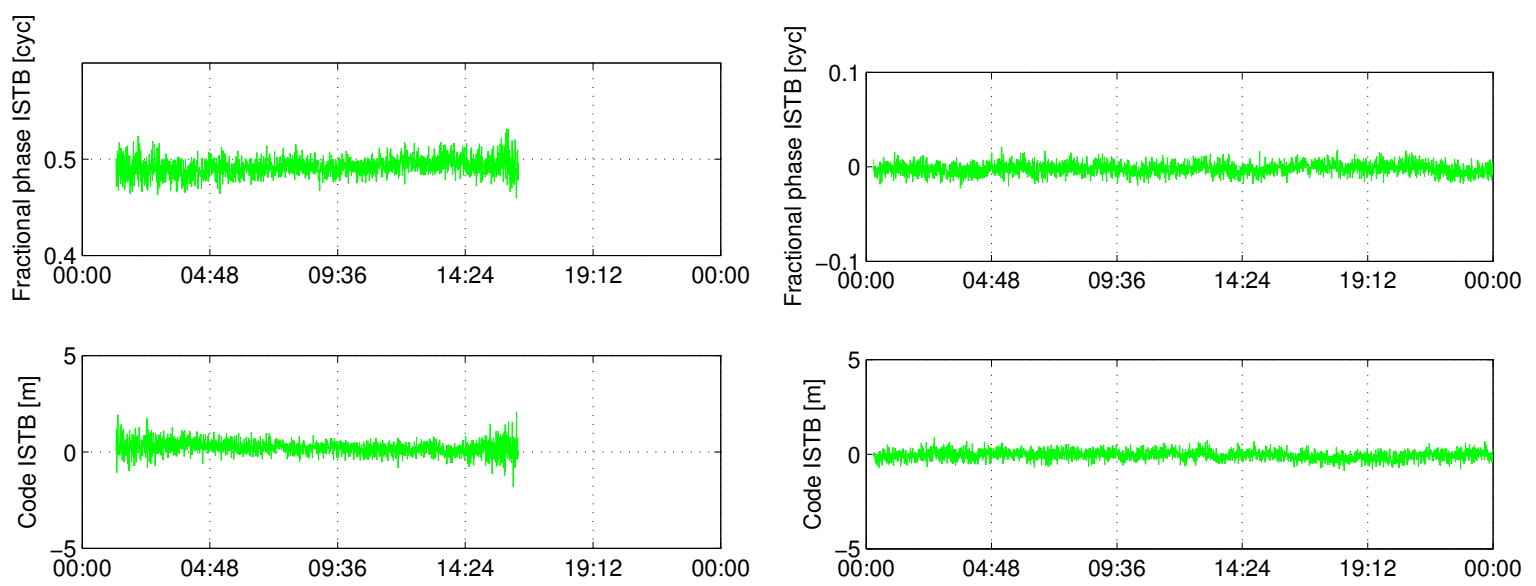

(c)

(d)
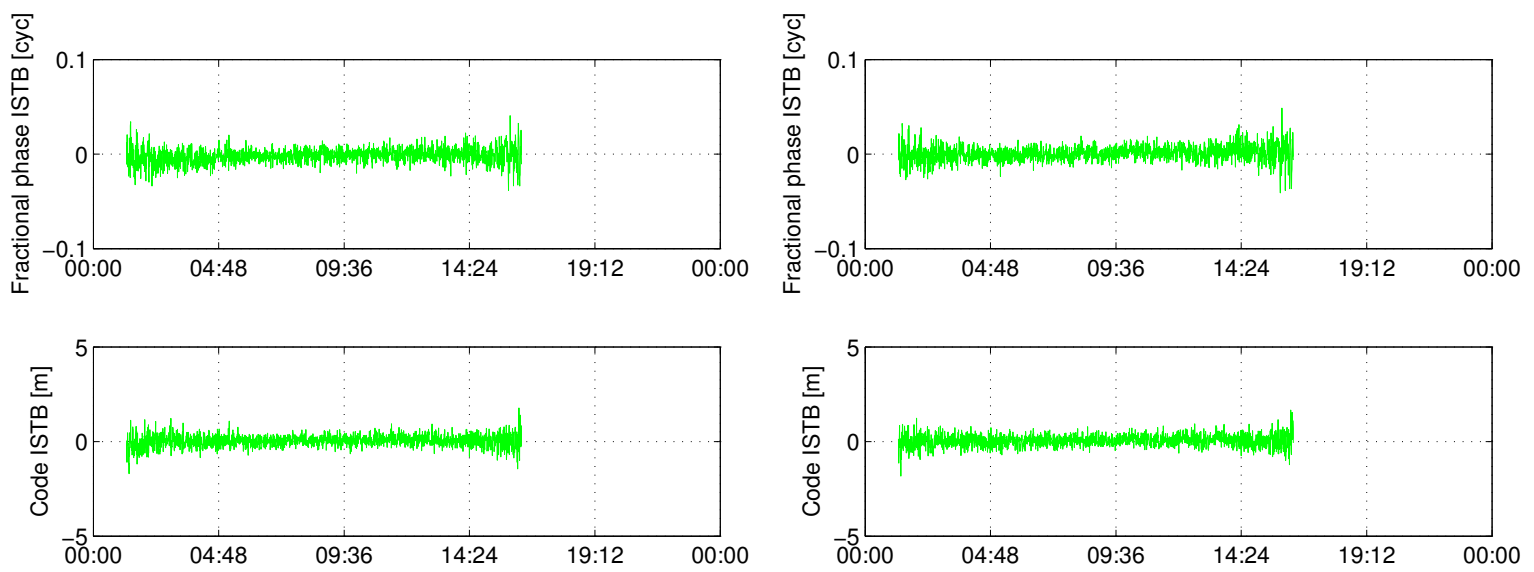

(e)

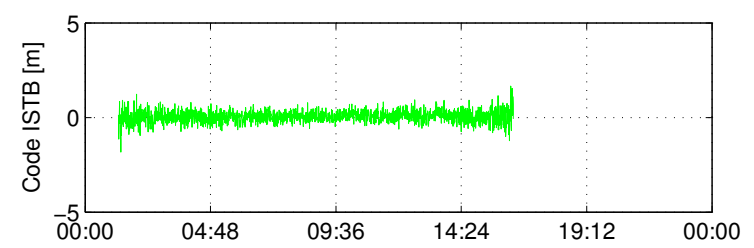

(f)

Table 5 and 6 summarize the ISTB estimates and their standard deviations, clearly indicating the existence of non-zero ISTBs (highlighted using bold text) between dissimilar receiver types. It was 
observed that the estimated ISTBs are constant for given receiver-antenna connectivity and receiver operating environment. Nevertheless, it was found that the observed code ISTBs do not significantly affect ambiguity resolution and the consequent ambiguity resolved phase-only baseline estimation. However, the phase ISTBs severely affect ambiguity resolution, especially in the case of half-cycle phase ISTBs. As summarized in Table 7, GEO satellites have phase ISTBs of half-cycles with respect to IGSO/MEO satellites in the case of mixed receivers. Note that phase ISTBs for other receiver pairs can be deduced from estimates in Table 7. For example, with the sign convention in Table 4, the Septentrio-Javad pair has phase ISTBs of -0.5 cycle and 0.5 cycle for B1 and B2, respectively. Note that, for attitude determination with the ISTB-corrected model in the following section, we only correct phase observations with half cycles, as other (code) biases were found to be small enough to not affect the ambiguity resolution significantly.

Table 5. Estimated ISTBs using five days of data from Curtin's zero baselines.

\begin{tabular}{|c|c|c|c|c|c|c|c|}
\hline \multirow[b]{2}{*}{ Receiver Pair } & \multirow[b]{2}{*}{ Frequency } & \multicolumn{2}{|c|}{ IGSO-GEO } & \multicolumn{2}{|c|}{ IGSO-MEO } & \multicolumn{2}{|c|}{ GEO-MEO } \\
\hline & & $\begin{array}{l}\text { Phase } \\
\text { ISTB/std } \\
\text { (cyc) }\end{array}$ & $\begin{array}{l}\text { Code ISTB/std } \\
\text { (m) }\end{array}$ & $\begin{array}{l}\text { Phase } \\
\text { ISTB/std } \\
\text { (cyc) }\end{array}$ & $\begin{array}{l}\text { Code ISTB/std } \\
\text { (m) }\end{array}$ & $\begin{array}{l}\text { Phase } \\
\text { ISTB/std } \\
\text { (cyc) }\end{array}$ & $\begin{array}{l}\text { Code ISTB/std } \\
\text { (m) }\end{array}$ \\
\hline CUT0-CUT1 & B1 & $0.00 / 0.000$ & $-\mathbf{0 . 0 7 / 0 . 0 0 2}$ & $0.00 / 0.000$ & $\mathbf{0 . 0 3 / 0 . 0 0 3}$ & $0.00 / 0.000$ & $\mathbf{0 . 1 0} / 0.003$ \\
\hline $\begin{array}{l}\text { (Trimble- } \\
\text { Septentrio) }\end{array}$ & B2 & $-\mathbf{0 . 5 0} / 0.000$ & $-\mathbf{0 . 0 1 / 0 . 0 0 2}$ & $0.00 / 0.000$ & $\mathbf{0 . 0 1} / 0.003$ & $\mathbf{0 . 5 0 / 0 . 0 0 0}$ & $\mathbf{0 . 0 2} / 0.003$ \\
\hline CUT0-CUT2 & B1 & $0.00 / 0.000$ & $0.00 / 0.002$ & $0.00 / 0.000$ & $0.00 / 0.003$ & $0.00 / 0.000$ & $0.00 / 0.003$ \\
\hline (Trimble-Trimble) & $\mathrm{B} 2$ & $0.00 / 0.000$ & $0.00 / 0.002$ & $0.00 / 0.000$ & $0.00 / 0.003$ & $0.00 / 0.000$ & $0.00 / 0.003$ \\
\hline CUT0-CUT3 & B1 & $-\mathbf{0 . 5 0} / 0.000$ & $-\mathbf{0 . 0 6 / 0 . 0 0 2}$ & $0.00 / 0.000$ & $\mathbf{0 . 0 4} / 0.003$ & $\mathbf{0 . 4 9} / 0.000$ & $\mathbf{0 . 1 0} / 0.003$ \\
\hline (Trimble-Javad) & $\mathrm{B} 2$ & $-0.00 / 0.000$ & $\mathbf{0 . 0 3 / 0 . 0 0 2}$ & $0.00 / 0.000$ & $\mathbf{0 . 0 5} / 0.003$ & $0.00 / 0.000$ & $\mathbf{0 . 0 1 / 0 . 0 0 4}$ \\
\hline CUTA-CUAA & B1 & $-\mathbf{0 . 5 0} / 0.000$ & $-\mathbf{0 . 0 6} / 0.002$ & $0.00 / 0.000$ & $\mathbf{0 . 0 1 / 0 . 0 0 3}$ & $\mathbf{0 . 5 0} / 0.000$ & $\mathbf{0 . 0 7 / 0 . 0 0 3}$ \\
\hline (Trimble-Javad) & B2 & $0.00 / 0.000$ & $\mathbf{0 . 0 4} / 0.002$ & $0.00 / 0.000$ & $\mathbf{0 . 0 3} / 0.003$ & $0.00 / 0.000$ & $-\mathbf{0 . 0 2} / 0.003$ \\
\hline
\end{tabular}

Table 6. Estimated ISTBs using data from zero baseline experiments with Trimble-Javad receiver pairs: two in Curtin University and another two in Kalamunda (Table 2).

\begin{tabular}{|c|c|c|c|c|c|c|c|}
\hline \multirow[b]{2}{*}{ Experiment } & \multirow[b]{2}{*}{ Frequency } & \multicolumn{2}{|c|}{ IGSO-GEO } & \multicolumn{2}{|c|}{ IGSO-MEO } & \multicolumn{2}{|c|}{ GEO-MEO } \\
\hline & & $\begin{array}{l}\text { Phase } \\
\text { ISTB/std } \\
\text { (cyc) }\end{array}$ & $\begin{array}{l}\text { Code ISTB/std } \\
\text { (m) }\end{array}$ & $\begin{array}{l}\text { Phase } \\
\text { ISTB/std } \\
\text { (cyc) }\end{array}$ & $\begin{array}{l}\text { Code ISTB/std } \\
\text { (m) }\end{array}$ & $\begin{array}{l}\text { Phase } \\
\text { ISTB/std } \\
\text { (cyc) }\end{array}$ & $\begin{array}{l}\text { Code ISTB/std } \\
\text { (m) }\end{array}$ \\
\hline \multirow{2}{*}{ Curtin 1} & B1 & $-\mathbf{0 . 5 0} / 0.000$ & $-0.17 / 0.003$ & $0.00 / 0.000$ & $\mathbf{0 . 0 6} / 0.005$ & $\mathbf{0 . 4 9 / 0 . 0 0 0}$ & $\mathbf{0 . 2 3} / 0.005$ \\
\hline & B2 & $0.00 / 0.000$ & $-0.06 / 0.003$ & $0.00 / 0.000$ & $\mathbf{0 . 0 4} / 0.005$ & $0.00 / 0.000$ & $\mathbf{0 . 1 0} / 0.005$ \\
\hline \multirow{2}{*}{ Curtin 2} & B1 & $-\mathbf{0 . 5 0} / 0.000$ & $-0.16 / 0.003$ & $0.00 / 0.000$ & $\mathbf{0 . 0 5 / 0 . 0 0 6}$ & $0.49 / 0.000$ & $\mathbf{0 . 2 0} / 0.006$ \\
\hline & B2 & $0.00 / 0.000$ & $-0.06 / 0.003$ & $0.00 / 0.000$ & $\mathbf{0 . 0 3} / 0.006$ & $0.00 / 0.000$ & $\mathbf{0 . 0 8} / 0.006$ \\
\hline \multirow{2}{*}{ Kalamunda 1} & B1 & $-\mathbf{0 . 5 0} / 0.000$ & $-\mathbf{0 . 1 2} / 0.003$ & $0.00 / 0.000$ & $\mathbf{0 . 0 4} / 0.005$ & $\mathbf{0 . 4 9 / 0 . 0 0 0}$ & $\mathbf{0 . 1 6} / 0.005$ \\
\hline & B2 & $0.00 / 0.000$ & $-\mathbf{0 . 0 3 / 0 . 0 0 3}$ & $0.00 / 0.000$ & $\mathbf{0 . 0 3 / 0 . 0 0 5}$ & $0.00 / 0.000$ & $\mathbf{0 . 0 6 / 0 . 0 0 5}$ \\
\hline \multirow{2}{*}{ Kalamunda 2} & B1 & $-\mathbf{0 . 5 0} / 0.000$ & $-\mathbf{0 . 1 1} / 0.003$ & $0.00 / 0.000$ & $\mathbf{0 . 0 4} / 0.005$ & $\mathbf{0 . 4 9} / 0.000$ & $\mathbf{0 . 1 6} / 0.005$ \\
\hline & B2 & $0.00 / 0.000$ & $-\mathbf{0 . 0 2} / 0.003$ & $0.00 / 0.000$ & $\mathbf{0 . 0 4} / 0.005$ & $0.00 / 0.000$ & $0.07 / 0.005$ \\
\hline
\end{tabular}


Table 7. Differential phase ISTB between GEO and IGSO/MEO satellites with Trimble as the pivot receiver (cyc) and based on the sign convention considered in Table 4

\begin{tabular}{lccc}
\hline Frequency & Trimble & Septentrio & Javad \\
\hline B1 & 0 & 0 & -0.5 \\
B2 & 0 & -0.5 & 0 \\
\hline
\end{tabular}

\subsection{Effect of ISTBs on Attitude Determination}

Next, we analysed the impact of ISTBs on BeiDou single- and dual-frequency instantaneous attitude determination using the standard LAMBDA and C-LAMBDA methods comparing three processing approaches, namely, the classical DD model with ignoring ISTBs in Equation (19), the extended model in Equation (26) and the classical DD model with ISTB correction in Equation (28). Results for different receiver pairs (CUT0-CUTA, CUT0-CUAA, CUT1-CUTA and CUT1-CUAA), which consist of mixed receivers forming a short baseline of $8.418 \mathrm{~m}$, as shown in Figure 1b, are discussed in the following. Note that these receiver pairs are not used in the computation of ISTBs in Section 4.2. We considered two performance measures for our analyses; the first one is the empirical instantaneous ambiguity success fraction (relative frequency), which is defined as:

$$
\text { success fraction }=\frac{\text { number of correctly fixed epochs }}{\text { total number of epochs }}
$$

where the true ambiguities are computed using known antenna coordinates in WGS84, as the antennas used are part of Curtin's permanent stations. However, only length information is used for C-LAMBDA processing. The second performance measure is the ambiguity fixed angular estimation accuracy, which is given by the formal and empirical standard deviations of attitude angular estimates.

Table 8-10 report the instantaneous ambiguity success fraction for single-frequency B1, single-frequency B2 and dual frequency B1-B2 processing, respectively. The first row in each table corresponds to the baseline with the same receiver type for which ISTBs are zero and correction is not needed. The third row in Table 8 and the second row in Table 9 correspond to baselines with dissimilar receiver types, which have zero ISTBs for corresponding frequencies. The benefits of using C-LAMBDA, which makes use of known baseline length, are highlighted using bold text. Furthermore, catastrophic failures of ambiguity resolution by ignoring non-zero ISTBs are highlighted with emphasized text. Hence, it is wise to use the extended model (or the type-specific DD model) if one does not have the knowledge of ISTB between dissimilar receiver pairs. However, the best processing strategy is to use ISTB-corrected classical double differencing. With ISTB calibration, the C-LAMBDA method yields single-frequency instantaneous attitude determination. 
Table 8. Instantaneous ambiguity success fractions (relative frequencies) for single-frequency (B1) processing.

\begin{tabular}{|c|c|c|c|c|c|c|}
\hline \multirow[t]{2}{*}{ Baseline } & \multicolumn{2}{|c|}{$\begin{array}{l}\text { Classical DD model Equa- } \\
\text { tion (19) }\end{array}$} & \multicolumn{2}{|c|}{$\begin{array}{l}\text { Extended model Equation } \\
\text { (26) }\end{array}$} & \multicolumn{2}{|c|}{$\begin{array}{l}\text { Classical DD model with } \\
\text { ISTB correction Equa- } \\
\text { tion }(28)\end{array}$} \\
\hline & LAMBDA & C-LAMBDA & LAMBDA & C-LAMBDA & LAMBDA & C-LAMBDA \\
\hline $\begin{array}{l}\text { CUT0-CUTA } \\
\text { (Trimble-Trimble) }\end{array}$ & 0.98 & 1.00 & 0.77 & 0.99 & 0.98 & 1.00 \\
\hline $\begin{array}{l}\text { CUT0-CUAA } \\
\text { (Trimble-Javad) }\end{array}$ & 0.00 & 0.00 & 0.75 & 0.99 & 0.97 & 1.00 \\
\hline $\begin{array}{l}\text { CUT1-CUTA } \\
\text { (Septentrio-Trimble) }\end{array}$ & 0.99 & 1.00 & 0.87 & 1.00 & 0.99 & 1.00 \\
\hline $\begin{array}{l}\text { CUT1-CUAA } \\
\text { (Septentrio-Javad) }\end{array}$ & 0.00 & 0.00 & 0.86 & 1.00 & 0.99 & 1.00 \\
\hline
\end{tabular}

Table 9. Instantaneous ambiguity success fractions (relative frequencies) for single-frequency (B2) processing.

\begin{tabular}{|c|c|c|c|c|c|c|}
\hline \multirow[t]{2}{*}{ Baseline } & \multicolumn{2}{|c|}{$\begin{array}{l}\text { Classical DD model Equa- } \\
\text { tion (19) }\end{array}$} & \multicolumn{2}{|c|}{$\begin{array}{l}\text { Extended model Equation } \\
\text { (26) }\end{array}$} & \multicolumn{2}{|c|}{$\begin{array}{l}\text { Classical DD model with } \\
\text { ISTB correction Equation } \\
\text { (28) }\end{array}$} \\
\hline & LAMBDA & C-LAMBDA & LAMBDA & C-LAMBDA & LAMBDA & C-LAMBDA \\
\hline $\begin{array}{l}\text { CUT0-CUTA } \\
\text { (Trimble-Trimble) }\end{array}$ & 0.98 & 1.00 & 0.86 & 1.00 & 0.98 & 1.00 \\
\hline $\begin{array}{l}\text { CUT0-CUAA } \\
\text { (Trimble-Javad) }\end{array}$ & 0.99 & 1.00 & 0.88 & 1.00 & 0.99 & 1.00 \\
\hline $\begin{array}{l}\text { CUT1-CUTA } \\
\text { (Septentrio-Trimble) }\end{array}$ & 0.00 & 0.00 & 0.94 & 1.00 & 0.99 & 1.00 \\
\hline $\begin{array}{l}\text { CUT1-CUAA } \\
\text { (Septentrio-Javad) }\end{array}$ & 0.00 & 0.00 & 0.96 & 1.00 & 1.00 & 1.00 \\
\hline
\end{tabular}

Finally, Table 11 reports ambiguity fixed angular accuracy for single- and dual-frequency processing. Since the baselines $(8.418 \mathrm{~m})$ considered in these analyses are formed by receivers with similar noise characteristics, the ambiguity fixed angular standard deviations are the same for all cases, except the cases with catastrophic failure of ambiguity resolution. Hence, we report the average angular standard deviation of all other cases. Single-frequency processing with either B1 or B2 yields the same angular accuracy. The improved dual-frequency angular accuracy reflects the increased redundancy. 
Table 10. Instantaneous ambiguity success fractions (relative frequencies) for dual-frequency (B1-B2) processing.

\begin{tabular}{|c|c|c|c|c|c|c|}
\hline \multirow[t]{2}{*}{ Baseline } & \multicolumn{2}{|c|}{$\begin{array}{l}\text { Classical DD model Equa- } \\
\text { tion (19) }\end{array}$} & \multicolumn{2}{|c|}{$\begin{array}{l}\text { Extended model Equation } \\
\text { (26) }\end{array}$} & \multicolumn{2}{|c|}{$\begin{array}{l}\text { Classical DD model with } \\
\text { ISTB correction Equa- } \\
\text { tion }(28)\end{array}$} \\
\hline & LAMBDA & C-LAMBDA & LAMBDA & C-LAMBDA & LAMBDA & C-LAMBDA \\
\hline $\begin{array}{l}\text { CUT0-CUTA } \\
\text { (Trimble-Trimble) }\end{array}$ & 1.00 & 1.00 & 1.00 & 1.00 & 1.00 & 1.00 \\
\hline $\begin{array}{l}\text { CUT0-CUAA } \\
\text { (Trimble-Javad) }\end{array}$ & 0.00 & 0.00 & 1.00 & 1.00 & 1.00 & 1.00 \\
\hline $\begin{array}{l}\text { CUT1-CUTA } \\
\text { (Septentrio-Trimble) }\end{array}$ & 0.00 & 0.00 & 1.00 & 1.00 & 1.00 & 1.00 \\
\hline $\begin{array}{l}\text { CUT1-CUAA } \\
\text { (Septentrio-Javad) }\end{array}$ & 0.00 & 0.00 & 1.00 & 1.00 & 1.00 & 1.00 \\
\hline
\end{tabular}

Table 11. Empirical and formal (given in brackets) angular standard deviation (deg).

\begin{tabular}{lccc}
\hline & Single-Frequency (B1) & Single-Frequency (B2) & Dual-Frequency (B1-B2) \\
\hline Heading & $0.02(0.02)$ & $0.02(0.02)$ & $0.01(0.01)$ \\
Elevation & $0.04(0.04)$ & $0.04(0.04)$ & $0.03(0.03)$ \\
\hline
\end{tabular}

\section{Summary and Conclusions}

In this contribution, we investigated the existence of BeiDou inter-satellite-type biases (ISTBs) and their impact on standalone BeiDou attitude determination with mixed receiver types. We considered an extended GNSS double difference model incorporating all possible differential ISTBs among the three BeiDou satellite types (GEO, IGSO and MEO), together with three processing approaches, namely, one based on the classical double differenced model, ignoring the ISTBs, another based on the extended double differenced model, incorporating the ISTBs, and a third one based on the ISTB-corrected classical double differenced model. Our analyses using two real data sets with three different receiver types demonstrate the existence of non-zero ISTBs between different satellite types. The estimated ISTBs are stable and can be used to correct mixed receiver BeiDou attitude determination. It was observed that the estimated ISTBs are constant for a given receiver-antenna connectivity and receiver operating environment. Nevertheless, it was found that the observed code ISTBs do not significantly affect ambiguity resolution and the consequent ambiguity resolved phase-only baseline estimation. However, the mixed receiver half-cycle phase ISTBs severely affect ambiguity resolution. This finding is an important warning for mixed receiver type users, including precise point positioning users [54,55,57,58]. Moreover, it may also trigger GNSS receiver manufacturers to develop mutually consistent measurement extractions, as they are in the early stage of BeiDou-enabled receiver developments. Furthermore, it is suggested to use the extended model or, equivalently, the type-specific DD model, if one does not have the knowledge of ISTBs between dissimilar receiver pairs. However, the best processing strategy is to 
use the ISTB-corrected classical double differencing procedure. With ISTB correction, the C-LAMBDA method enables single-frequency, instantaneous attitude determination capability in the Asia-Pacific region with the current BeiDou constellation.

\section{Acknowledgments}

This work is supported by the Australian Space Research Program GARADA project on SAR Formation Flying. The second author, P.J.G. Teunissen, is the recipient of an Australian Research Council Federation Fellowship (project number FF0883188). All this support is gratefully acknowledged.

\section{Conflict of Interest}

The authors declare no conflict of interest.

\section{References}

1. CSNO. BeiDou Navigation Satellite System Signal in Space Interface Control Document: Open Service Signal B1I; Version 1.0. China Satellite Navigation Office: Beijing, China, December 2012.

2. Cao, C.; Jing, G.; Luo, M. COMPASS Satellite Navigation System Development. PNT Challenges and Opportunities Symposium, Stanford, CA, USA, 5-6 November 2008.

3. Grelier, T.; Ghion, A.; Dantepal, J.; Ries, L.; de Latour, A.; Issler, J.L.; Avila-Rodriguez, J.; Wallner, S.; GW, H. COMPASS signal structure and first measurements. ION GNSS 2007, 2007, 3015-3024.

4. Chen, H.; Huang, Y.; Chiang, K.; Yang, M.; Rau, R. The performance comparison between GPs and BeiDou-2/COMPASS: A perspective from Asia. J. Chin. Inst. Eng. 2009, 32, 679-689.

5. Yang, Y.; Li, J.; Xu, J.; Tang, J.; Guo, H.; He, H. Contribution of the Compass satellite navigation system to global PNT users. Chin. Sci. Bull. 2011, 56, 2813-2819.

6. Zhang, S.; Guo, J.; Li, B.; Rizos, C. An Analysis of Satellite Visibility and Relative Positioning Precision of COMPASS. In Proceedings of Symposium for Chinese Professionals in GPS, Shanghai, China, 18-20 August 2011. pp. 41-46.

7. Verhagen, S.; Teunissen, P.J. Ambiguity resolution performance with GPS and BeiDou for LEO formation flying. Adv. Space Res. 2013, doi: 10.1016/j.asr.2013.03.007.

8. Montenbruck, O.; Hauschild, A.; Steigenberger, P.; Hugentobler, U.; Teunissen, P.J.G.; Nakamura, S. Initial assessment of the COMPASS/BeiDou-2 regional navigation satellite system. GPS Solut. 2013, 17, 211-222.

9. Montenbruck, O.; Hauschild, A.; Steigenberger, P.; Hugentobler, U.; Riley, S. A COMPASS for Asia: First Experience with the BeiDou-2 Regional Navigation System. IGS Workshop, Olsztyn, Poland, 23-27 July 2012.

10. Shi, C.; Zhao, Q.; Li, M.; Tang, W.; Hu, Z.; Lou, Y.; Zhang, H.; Niu, X.; Liu, J. Precise orbit determination of BeiDou Satellites with precise positioning. Sci. China Earth Sci. 2012, 55, 1079-1086. 
11. Steigenberger, P.; Hauschild, A.; Montenbruck, O.; Hugentobler, U. Performance Analysis of COMPASS Orbit and Clock Determination and COMPASS-only PPP. IGS Workshop, Olsztyn, Poland, 23-27 July 2012.

12. Odolinski, R.; Teunissen, P.J.G.; Odijk, D. An Analysis of Combined COMPASS/BeiDou-2 and GPS Single- and Multiple-Frequency RTK Positioning. In Proceedings of the Institute of Navigation Pacific PNT 2013, Honolulu, HI, USA, 22-25 April 2013; pp. 69-90.

13. Cai, C.; Gao, Y.; Pan, L.; Dai, W. An analysis on combined GPS/COMPASS data quality and its effect on single point positioning accuracy under different observing conditions. Adv. Space Res. 2013, doi:10.1016/j.asr.2013.02.019.

14. Li, W.; Teunissen, P.J.G.; Zhang, B.; Verhagen, S. Precise Point Positioning Using GPS and Compass Observations. In Proceedings of the 4th China Satellite Navigation Conference (CSNC), Wuhan, China, 15-17 May 2013.

15. Zhao, Q.; Guo, J.; Li, M.; Qu, L.; Hu, Z.; Shi, C.; Liu, J. Initial results of precise orbit and clock determination for COMPASS navigation satellite system. J. Geod. 2013, 87, 475-486.

16. Nadarajah, N.; Teunissen, P.J.G.; Buist, P.; Steigenberger, P. First Results of Instantaneous GPS/Galileo/COMPASS Attitude Determination. In Proceedings of the 6th ESA Workshop on Satellite Navigation User Equipment Technologies, Noordwijk, The Netherlands, 5-7 December 2012; p. 8.

17. Nadarajah, N.; Teunissen, P.J.G. Instantaneous GPS/BeiDou/Galileo Attitude Determination: A Single-Frequencyr Robustness Analysis under Constrained Environments. In Proceedings of The Institute of Navigation Pacific PNT, Honolulu, HI, USA, 22-25 April, 2013; pp. 1088-1103.

18. Nadarajah, N.; Teunissen, P.J.G.; Raziq, N. Instantaneous COMPASS-GPS attitude determination: A robustness analysis. Adv. Space Res. 2013, submitted.

19. Shi, C.; Zhao, Q.; Hu, Z.; Liu, J. Precise relative positioning using real tracking data from COMPASS GEO and IGSO satellites. GPS Solut. 2013, 17, 103-119.

20. Steigenberger, P.; Hauschild, A.; Montenbruck, O.; Rodriguez-Solano, C.; Hugentobler, U. Orbit and clock determination of QZS-1 based on the CONGO network. ION-ITM-2012, 2012.

21. Steigenberger, P.; Hugentobler, U.; Hauschild, A.; Montenbruck, O. Orbit and clock analysis of Compass GEO and IGSO satellites. J. Geod. 2013, 87, 515-525.

22. Cohen, C. Attitude Determination Using GPS. Ph.D. Thesis, Stanford University, Stanford, CA, USA, 1992.

23. Lu, G. Development of A GPS Multi-Antenna System for Attitude Determination. Ph.D. Thesis, University of Calgary, Calgary, Alberta, Canada, 1995.

24. Crassidis, J.L.; Markley, F.L. New algorithm for attitude determination using Global Positioning System signals. J. Guidance Control Dyn. 1997, 20, 891-896.

25. Li, Y.; Zhang, K.; Roberts, C.; Murata, M. On-the-fly GPS-based attitude determination using single- and double-differenced carrier phase measurements. GPS Solut. 2004, 8, 93-102.

26. Lin, D.; Voon, L.; Nagarajan, N. Real-time Attitude Determination for Microsatellite by LAMBDA Method Combined with Kalman Filtering. In Proceedings of the 22nd AIAA International Communications Satellite Systems Conference and Exhibit, Monterey, California, USA, 9-12 May 2004. 
27. Madsen, J.; Lightsey, E.G. Robust spacecraft attitude determination using global positioning system receivers. J. Spacecr. Rocket. 2004, 41, 635-643.

28. Psiaki, M.L. Batch algorithm for global-positioning-system attitude determination and integer ambiguity resolution. J. Guidance Control Dyn. 2006, 29, 1070-1079.

29. Teunissen, P.J.G. The least-squares ambiguity decorrelation adjustment: A method for fast GPS integer ambiguity estimation. J. Geod. 1995, 70, 65-82.

30. Boon, F.; Ambrosius, B. Results of Real-time Applications of the LAMBDA Method in GPS Based Aircraft Landings. In Proceedings of the International Symposium on Kinematic Systems in Geodesy, Geomatics and Navigation, Banff, AB, Canada, 3-6 June 1997; pp. 339-345.

31. Cox, D.B.; Brading, J.D.W. Integration of LAMBDA Ambiguity Resolution with Kalman Filter for Relative Navigation of Spacecraft. In Proceedings of the 55th Annual Meeting of The Institute of Navigation, Cambridge, MA, USA, 27-30 June, 1999; pp. 739-745.

32. Ji, S.; Chen, W.; Zhao, C.; Ding, X.; Chen, Y. Single epoch ambiguity resolution for Galileo with the CAR and LAMBDA methods. GPS Solut. 2007, 11, 259-268.

33. Huang, S.; Wang, J.; Wang, X.; Chen, J. The application of the LAMBDA method in the estimation of the GPS slant wet vapour. Acta Astron. Sinica 2009, 50, 60-68.

34. Kroes, R.; Montenbruck, O.; Bertiger, W.; Visser, P. Precise GRACE baseline determination using GPS. GPS Solutions 2005, 9, 21-31.

35. Jin, S.; Luo, O.; Ren, C. Effects of physical correlations on long-distance GPS positioning and zenith tropospheric delay estimates. Adv. Space Res. 2010, 46, 190-195.

36. Jin, S.; Wang, J.; Park, P.H. An improvement of GPS height estimations-Stochastic modeling. Earth Planets Space 2005, 57, 253-259.

37. Park, S.Y. Thermally induced attitude disturbance control for spacecraft with a flexible boom. J. Spacecr. Rocket. 2002, 39, 325-328.

38. Teunissen, P.J.G. An optimality property of the integer least-squares estimator. J. Geod. 1999, 73, 587-593.

39. Teunissen, P.J.G.; De Jonge, P.; Tiberius, C. Performance of the LAMBDA method for fast GPS ambiguity resolution. Navigation 1997, 44, 373-383.

40. Verhagen, S.; Teunissen, P.J.G. New global navigation satellite system ambiguity resolution method compared to existing approaches. J. Guidance Control Dyn. 2006, 29, 981-991.

41. Park, C.; Teunissen, P.J.G. A New Carrier Phase Ambiguity Estimation for GNSS Attitude Determination Systems. In Proceedings of International Symposium on GPS/GNSS, Tokyo, Japan, 15-18 November 2003; pp. 283-290.

42. Teunissen, P.J.G. The LAMBDA method for the GNSS compass. Artif. Satell. 2006, 41, 89-103.

43. Buist, P.J. The Baseline Constrained LAMBDA Method for Single Epoch, Single Frequency Attitude Determination Applications. In Proceedings of the 20th International Technical Meeting of the Satellite Division of the Institute of Navigation (ION GNSS 2007), Fort Worth, TX, USA, 25-28 September 2007; pp. 2962-2973.

44. Park, C.; Teunissen, P.J.G. Integer least squares with quadratic equality constraints and its application to GNSS attitude determination systems. Int. J. Control Autom. Syst. 2009, 7, 566-576. 
45. Giorgi, G.; Teunissen, P.J.G.; Buist, P.J. A Search and Shrink Approach for the Baseline Constrained LAMBDA Method: Experimental Results. In Proceedings of International Symposium on GPS/GNSS, Tokyo, Japan, 11-14 November 2008; pp. 797-806.

46. Giorgi, G.; Buist, P. Single-epoch, Single-frequency, Standalone Full Attitude Determination: Experimental Results. In Proceedings of the 4th ESA Workshop on Satellite Navigation User Equipment Technologies, NAVITEC, Noordwijk, The Netherlands, 10-12 December 2008; p. 8.

47. Teunissen, P.J.G. Integer least-squares theory for the GNSS compass. J. Geod. 2010, 84, 433-447.

48. Giorgi, G.; Teunissen, P.J.G.; Verhagen, S.; Buist, P.J. Testing a new multivariate GNSS carrier phase attitude determination method for remote sensing platforms. Adv. Space Res. 2010, 46, 118-129.

49. Teunissen, P.J.G.; Giorgi, G.; Buist, P.J. Testing of a new single-frequency GNSS carrier phase attitude determination method: Land, ship and aircraft experiments. GPS Solut. 2011, 15, 15-28.

50. Teunissen, P.J.G.; Kleusberg, A. GPS for Geodesy, 2nd ed.; Springer: Berlin/Heidelberg, Germany, 1998.

51. Harville, D.A. Matrix Algebra From A Statistician's Perspective; Springer: New York, NY, USA, 1997.

52. Magnus, J.R.; Neudecker, H. Matrix Differential Calculus with Applications in Statistics and Econometrics; Wiley: New York, NY, USA, 1995.

53. Teunissen, P.J.G. Zero Order Design: Generalized Inverses, Adjustment, the Datum Problem and S-transformations. In Optimization and Design of Geodetic Networks; Grafarend, E., Sans, F., Eds.; Springer: Berlin/Heidelberg, Germany, 1985; pp. 11-55.

54. Teunissen, P.J.G.; Odijk, D.; Zhang, B. PPP-RTK: Results of CORS network-based PPP with integer ambiguity resolution. J. Aeronaut. Astronaut. Aviat. Series A 2010, 42, 223-230.

55. Odijk, D.; Teunissen, P.J.G.; Zhang, B. Single-frequency integer ambiguity resolution enabled precise point positioning. J. Surv. Eng. 2012, 138, 193-202.

56. Euler, H.J.; Goad, C. On optimal filtering of GPS dual frequency observations without using orbit information. J. Geod. 1991, 65, 130-143.

57. Geng, J.; Meng, X.; Dodson, A.; Teferle, F. Integer ambiguity resolution in precise point positioning: Method comparison. J. Geod. 2010, 84, 569-581.

58. Zhang, B.; Teunissen, P.J.G.; Odijk, D. A novel un-differenced PPP-RTK concept. J. Navig. 2011, 64, S180-S191.

(c) 2013 by the authors; licensee MDPI, Basel, Switzerland. This article is an open access article distributed under the terms and conditions of the Creative Commons Attribution license (http://creativecommons.org/licenses/by/3.0/). 